\title{
Wayfinding on Foot From Information in Retinal, Not Optical, Flow
}

\author{
James E. Cutting \\ Cornell University
}

\author{
Ken Springer \\ Southern Methodist University
}

\author{
Paul A. Braren and Scott H. Johnson \\ Cornell University
}

\begin{abstract}
People find their way through cluttered environments with ease and without injury. How do they do it? Two approaches to wayfinding are considered: Differential motion parallax (DMP) is a retinal motion invariant of near and far objects moving against fixation; the information in optical flow (IOF) is a radial pattern of vectors, relying on decomposition of retinal flow. Evidence is presented that DMP guides wayfinding during natural gait, accounting for errors as well as correct responses. Evidence against IOF is also presented, and a space-time aliasing artifact that can contaminate IOF displays is explored. Finally, DMP and IOF are separated, showing they can yield different results in different environments. Thus, it is concluded that (a) DMP and IOF are different, (b) DMP and not IOF is used for wayfinding, (c) moving observers do not usually decompose retinal flow, and (d) optical flow may be a mathematical fiction with no psychological reality.
\end{abstract}

One of the most compelling of all visual phenomena occurs when one hurtles through the environment. The resulting radial streams of motion by surrounding objects, sometimes called optical flow, have captured the imagination of writers, artists, and cinematographers, as well as psychologists, neuroscientists, and computer scientists. This global motion was probably first noticed by the general populace in the mid19 th century, but then only in industrializing nations and with the widespread use of railroads.

The reasons for the relatively recent focus on optical flow are probably threefold: On a train one could, for the first time, travel (a) at velocities greater than about 4 eye heights/ $s$ for a sustained period of time; ${ }^{1}$ (b) on a relatively smooth roadbed that eliminated the bouncing caused by one's own footfall or that of a horse, or by the jostling of a coach; and (c) with free time to look about, unfettered by the demands of guiding one's course through the environment. Naturally, railway travel offered much more than noticeable optical flow. Not all of it was good. Indeed, around 1860 The Lancet published a series of articles on putative ill effects of rail travel

This research was supported by National Science Foundation Grant BNS-8818971 to James E. Cutting.

Experiment 1 was reported at the 30th Annual Meeting of the Psychonomic Society, Atlanta, November 1989; parts of Experiments 2 and 3 at the Society's 31 st Annual Meeting, New Orleans, November 1990; and parts of Experiments 7 and 8 at its 32nd Annual Meeting, San Francisco, November 1991. Experiments 1, 2, 4, 5, and 6 were also reported at a perception-action workshop at Storrs, Connecticut, in July 1990.

We thank Laurence G. Kaplan for initial and sustaining help in programming the Personal Iris Workstation; David Sabean for discussions of 19th-century perception; Gretchen Van de Walle, Jack Loomis, and several anonymous reviewers for their comments; and Julian Hochberg and Nan Karwan for various discussions of this project over the past 6 years.

Correspondence concerning this article should be addressed to James E. Cutting, Department of Psychology, Uris Hall, Cornell University, Ithaca, New York 14853-7601.
(Schivelbusch, 1986). Major clinical problems reported were eye fatigue, back strain, and overall body stress. For our purposes the causes of the first are the most interesting.

The railway journey forced a new way of looking at things, indeed perhaps even a novel way of perceiving space. In his studies of new perceptual niches created by industrialization, Schivelbusch (1986) described the situation of a passenger in a train compartment: ${ }^{2}$

Enclosed in it, the traveler has no way of distancing himself from the [nearby] objects [outside]-all he can do is to ignore them and the portions of the landscape that are closest to him, and to direct his gaze on the more distant objects that seem to pass by more slowly. If he does not modify his old way of observing things while traveling-if he still tries to perceive proximity and distance in equal measure-the result ... is fatigue. (p. 56) ... The [train's] speed causes objects to escape from one's gaze, but one nevertheless keeps trying to grasp them. (p. 57)

This "new way" of looking--following objects generally in the distance with one's eyes as near objects stream by, then saccading against the rapid flow to another relatively distant object-is an exaggerated form of the centerpiece of our article. We claim that, because of the new speeds attainable,

\footnotetext{
'The phenomenon of interest here is what might be called observer-scaled velocity, not velocity itself. What is important is the number of eye heights traveled per second, not meters per second. Consider the following: visual velocities are very slow when traveling in a jet at $900 \mathrm{~km} / \mathrm{hr}$ at an altitude of $10,000 \mathrm{~m}$; textures on the ground creep by the observer. Visual velocities are fast, however, when traveling in a sports car at $90 \mathrm{~km} / \mathrm{hr}$ with an eye height of $1 \mathrm{~m}$. In terms of observer-scale velocity this makes sense; in a plane an observer moves at about 0.025 eye heights/s and in a sports car he or she moves at about 25 eye heights/s.

${ }^{2}$ Schivelbusch $(1986,1988)$ has also explored the perceptual consequences of glass, candles, and incandescent lighting. Incandescent lighting made common for the first time new arrays of shadows, reflections, specularities, glint, and other perceptual phenomena, which we now take for granted.
} 
the railway journey intensified (and perhaps aggravated) this evolutionarily important strategy for people to find their way through everyday surroundings.

\section{Optical Flow, Retinal Flow, and Wayfinding}

More technically, optical flow is the relative motion of stationary objects around a moving observer. For linear travel, it is defined by the radial streams of motion and is seen best, if not exclusively, by an individual moving quite rapidly through an environment, well above pedestrian speeds. Most important, for our purposes, eye movements are ignored in optical flow.

Eye movements during locomotion generate much more complex, swirling patterns of motion, called retinal flow,

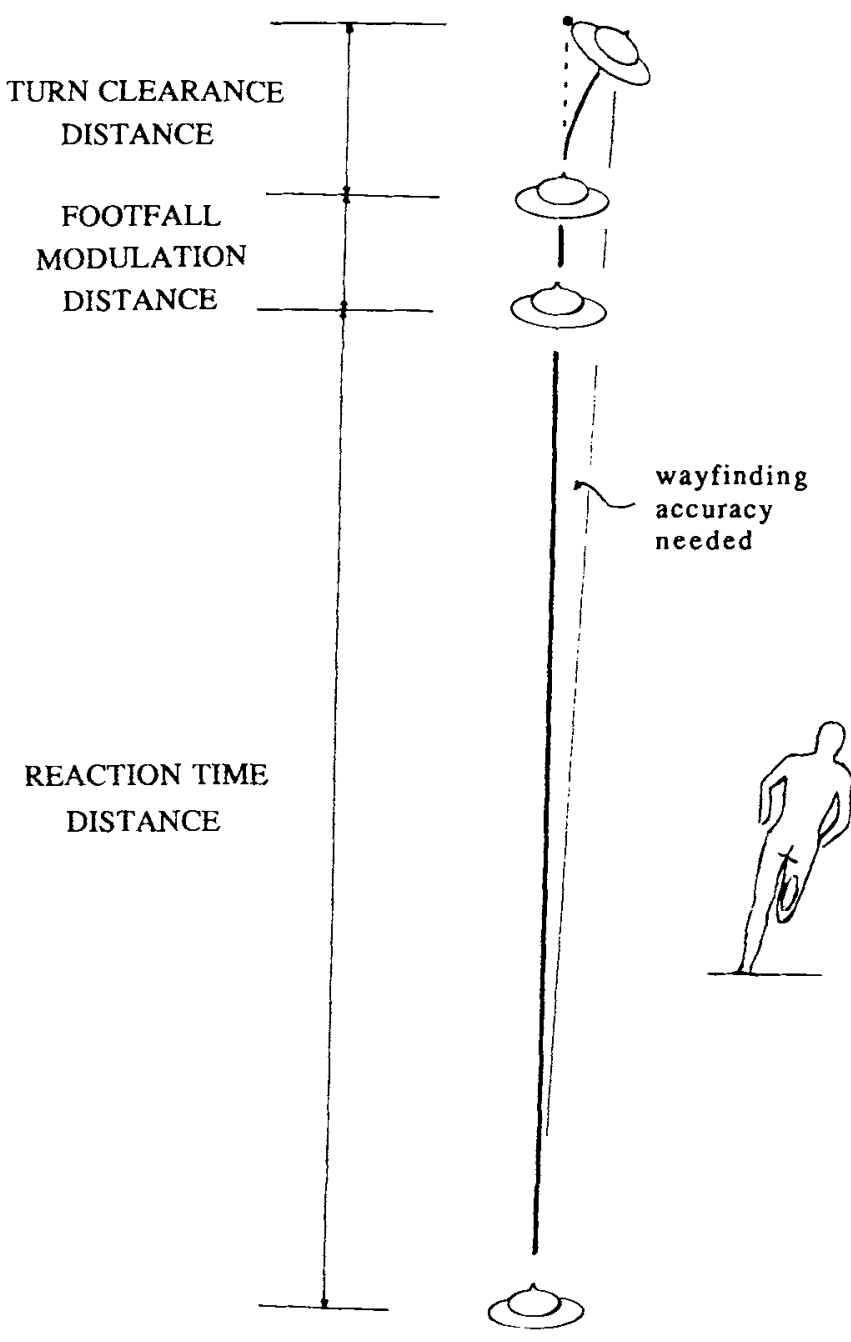

Figure 1. The distance components relevant to avoiding obstacles while moving through a cluttered environment. (Distances are scaled for a run of $6 \mathrm{~m} / \mathrm{s}$. Reaction time distance, based on a 3-s interval, far outstrips the other two distances in constraining the ability to avoid obstacles while moving through the environment. Experiment 3 corroborates the assumption of a 3-s reaction time interval. Also shown is a runner turning with a lean of $25^{\circ}$.) which are painted on the back of the eye. ${ }^{3}$ Until now, systematic study of global retinal flow has never been seriously considered, due largely to the influence of J. J. Gibson (1950, 1966,1979 ). We present a new view based on retinal flow in part because we feel most theoretical accounts of the motion information available to moving observers have been overly impressed by high-speed travel (e.g., by rail) and by Gibson's representations of optical motions. By comparison, the motion available to a pedestrian is quite slow.

There are many important perceptual phenomena associated with discussions of motion seen by moving observers $(\mathrm{R}$. Warren, 1990). One is the induced perception of self-motion, sometimes called vection (e.g., Andersen \& Braunstein, 1985; Dichgans \& Brandt, 1978; Johansson, 1977; Larish \& Flach, 1990; Lee \& Lishman, 1977; Rock \& Smith, 1986; Stoffregen, 1985). Large-scale, coherent motions can give the observer the impression he or she is moving, even if he or she is not. The railway journey probably gave birth to this phenomenon, and indeed, Duncker's (1912/1938) original discussion of induced motion-a phenomenon not usually associated with the perception of self-motion-begins with the effects of looking out from a stationary train while another moves slowly past.

The other important phenomenon, and the concern of this article, is wayfinding - the ability to find one's way through and around objects in a cluttered environment. The subtasks of wayfinding are to discern one's instantaneous course ${ }^{4}$ or aimpoint; and to negotiate clutter, while maintaining adequate velocity. We concentrate on the first subtask. How accurate must aimpoint determination be? To answer this question it seems best to consider a situation plausibly connected to the constraints under which we evolved.

\section{Wayfinding Accuracies Needed for Obstacle Avoidance}

Consider an individual running through a savannah or open forest, as suggested in Figure 1. The reason for running might be to escape an aggressor, to capture prey, or to bring news of a distant event. During the run, obstacles loom and must be avoided while maintaining footspeed. The act of obstacle avoidance can be parsed into three sequential time periods-

\footnotetext{
${ }^{3}$ By retinal flow, we mean the motion as it would be presented to the back of the eye. We are ignoring refractive error, the retinal mosaic, differential sensitivities to motion across the retina, the size of the field of view, and other attributes of and mechanisms in the eye itself. Retinal flow as we conceive it contrasts with optical flow only in that the orientation of the sensor (the eye) is anchored to the location of an external object, rather than remaining anchored in absolute coordinates.

${ }^{4}$ In much of the wayfinding literature the word heading is used to designate the aimpoint. In nautical and aeronautical circles, however, heading designates the direction a vehicle is pointing. Because of crosscurrents or crosswinds, however, vehicles crab (slip sideways) and move in a direction different than where they point. This direction is called the course or track in flying and bearing in sailing. Because of the nonintuitiveness of the terms course, track, and bearing, and because of potential confusions over the term heading, we use, following Ahumada (1983), the term aimpoint throughout.
} 
the reaction time necessary to recognize that an object is in one's path, the time necessary to adjust footfall, and the time necessary to negotiate a turn and avoid an object. Each of these periods has a distance associated with it, depending on forward velocity, and each of these distances constrains the tolerance limit for wayfinding. Let us start with the turn.

\section{Time and Distance to Negotiate a Turn}

Turning is constrained by physics. Generating a turn involves equalization of two forces. One stems from inertia, the tendency that keeps an already moving individual in motion toward, for instance, a tree. Because the tree is to be avoided while footspeed is maintained, the individual must adopt a curving path around the tree. For any circular motion the force associated with inertia $\left(F_{i}\right)$ is

$$
F_{i}=m v^{2} / r
$$

where $m$ is the mass of the individual, $v$ is the velocity, and $r$ is the radius of curvature for the path taken. Orthogonal to inertia is the turn-generating, or centripetal, force $\left(F_{c}\right)$. For a runner this is accomplished by leaning into the direction of the turn. The angle of lean $(\alpha)$ determines the radius of the turn, and the maximum angle of lean is determined by the coefficient for friction between bare foot and turf on a flat terrain. The centripetal force is

$$
F_{c}=\sin \alpha m g
$$

where the new term, $g$, is the gravitational constant (or 9.8 $\left.\mathrm{m} / \mathrm{s}^{2}\right)$.

Successful turns occur when these two forces are equal. If $F_{i}>F_{c}$, the individual will slide or stumble forward and fall, perhaps into the tree, causing injury or worse; if, on the other hand, $F_{i}<F_{c}$, then the individual will fall sideways and lose time or be overtaken by an aggressor. Thus, the right-hand sides of Equations 1 and 2 must be equal: $\sin \alpha m g=m v^{2} / r$. Simplifying and rearranging terms yields to focus on the radius of the turn produces

$$
r=v^{2} / \sin \alpha g,
$$

where masses cancel and only velocity, lean, and gravity remain.

Let us now assume that the individual is running between $2 \mathrm{~m} / \mathrm{s}$ and $10 \mathrm{~m} / \mathrm{s}$. The former is a slow jog and the latter is near the current world-record time for the $100-\mathrm{m}$ dash. Assume further that this individual can sustain a lean of $25^{\circ}$. The radius of curvature for the turn of avoidance, suggested in Figure 1, is between $1.0 \mathrm{~m}$ and $24.1 \mathrm{~m}$. To negotiate the turn successfully, the individual must lean and plant his or her foot, thrust sideways, and return upright, having moved laterally a leaning half-body width, or about $0.5 \mathrm{~m}$. This is the clearance. The most important subsequent calculation derived from this situation is the distance to the tree along the tangent to the circular path where the turn must begin. That distance, which we call the turn-clearance distance, depends on velocity and, given the velocity range above lies between $1.1 \mathrm{~m}$ and $5.0 \mathrm{~m}$, as shown in Table 1 . Now consider the beginning of the avoidance maneuver.
Table I

\begin{tabular}{|c|c|c|c|c|c|c|}
\hline \multirow[b]{2}{*}{ Wayfinding variable } & \multicolumn{6}{|c|}{ Velocity $(\mathrm{m} / \mathrm{s})$} \\
\hline & 10 & 6 & 5 & 4 & 3 & 2 \\
\hline $\begin{array}{l}\text { Required wayfinding accu- } \\
\text { racy (degrees) }\end{array}$ & 0.75 & 1.25 & 1.50 & 1.86 & 2.45 & 3.67 \\
\hline Turn clearance distance $(\mathrm{m})$ & 5.0 & 3.0 & 2.5 & 2.0 & 1.6 & 1.1 \\
\hline $\begin{array}{l}\text { Reaction time distance } \\
\quad(\mathrm{m} \text { in } 3.0 \mathrm{~s})\end{array}$ & 30.0 & 18.0 & 15.0 & 12.0 & 9.0 & 6.0 \\
\hline $\begin{array}{l}\text { Footfall modulation distance } \\
\quad(\mathrm{m} \text { in } 0.35 \mathrm{~s})\end{array}$ & 3.5 & 2.1 & 1.8 & 1.4 & 1.1 & 0.7 \\
\hline Total distance $(\mathrm{m})$ & 38.5 & 23.1 & 19.3 & 15.4 & 11.7 & 7.8 \\
\hline
\end{tabular}

Forward Velocities, Angular Requirements, and Component Distances for Wayfinding

Note. Ten meters per second is near the current world-record time for the $100-\mathrm{m}$ dash, $6 \mathrm{~m} / \mathrm{s}$ is near the current world-record marathon time (most humans at some time in their lives could sustain this pace, at least for brief periods), and $2 \mathrm{~m} / \mathrm{s}$ is a slow jog. To obtain wayfinding accuracies, divide the turn clearance $(0.5 \mathrm{~m})$ by the total distance and take the arctangent. The components making up the total distance are shown in Figure 1.

\section{Reaction Time and Distance Traveled}

On the basis of psychological experiments, it may seem appropriate to assume a reaction time of about $500 \mathrm{~ms}$ or less. This, however, would be foolhardy. In real-life situations, reaction times burgeon to $1.5 \mathrm{~s}$ and even $3.0 \mathrm{~s}$ due to clutter and multitask demands (Probst, Krafczyk, Brandt, \& Wist, 1984; Road Research Laboratory, 1963). With a footspeed between $2 \mathrm{~m} / \mathrm{s}$ and $10 \mathrm{~m} / \mathrm{s}$, the distance traveled during a reaction-time interval of $3 \mathrm{~s}$, which we call reaction-time distance, is between $6 \mathrm{~m}$ and $30 \mathrm{~m}$. One can begin to see that the requirements for wayfinding are largely determined by reaction time. The results of Experiment 3 reinforce our assumption of a 3-s reaction time interval.

\section{Footfall Regulation Time and Distance Traveled}

Finally, one cannot assume that the turn can be initiated exactly at the end of the reaction time interval-the runner's feet may not be on the ground. Moreover, one is usually constrained to initiate a turn on the outside foot; for example, for a right turn one must plant the left foot. Given that a step cycle (two steps) takes about $700 \mathrm{~ms}$ when running (Lee, Lishman, \& Thomson, 1982), an average of $350 \mathrm{~ms}$ must elapse before a right turn could begin. This adds $0.7 \mathrm{~m}$ to 3.5 $\mathrm{m}$ of footfall modulation distance.

\footnotetext{
${ }^{5}$ A $25^{\circ}$ lean is inadequate for many sports activities, which is why cleats and nubber-soled shoes were invented. In addition, we have assumed that a continuous circular turn is a good approximation for a step-by-step, discontinuous turn. This is true for a run, but less so for a walk. For a range of slower velocities, Patla, Prentice, Robinson, and Neufield (1991) assumed a discrete angled turn, but their results yield little that is different than what is assumed here. Finally, these calculations differ from those reported in Cutting (1986, pp. 277278 ) because different assumptions, particularly those about reaction time and clearance, have been made.
} 


\section{Accuracy Needed and a Rule of Thumb}

To determine the aimpoint accuracy needed, then, we add the reaction time, footfall modulation, and turning distances, then take the arctangent of the clearance over this total distance. For the extremes we have considered, the required accuracies are, for $2 \mathrm{~m} / \mathrm{s}$, atan $(0.5 / 7.8)=3.7^{\circ}$, and for 10 $\mathrm{m} / \mathrm{s}, \operatorname{atan}(0.5 / 38.5)=0.7^{\circ}$.

The upper limit, about $1^{\circ}$ accuracy, is interesting. Despite changes in velocities and coefficients of friction, about the same degree of accuracy is required for driving a car or skiing downhill. It is also needed to land an airplane (e.g., Hasbrook, 1975). Across the four types of locomotion-running, driving, skiing, and flying--one can see that technology and recreation have converged with evolution on this same important perceptual capacity.

For our purposes, however, we are concerned with accuracies in the $2^{\circ}-4^{\circ}$ range, the range for modest runs and fast walks. As a person's thumb held at arm's length subtends about $2^{\circ}$ visual angle, its width is a good approximation for the wayfinding accuracy needed: It is a true rule of thumb.

\section{Retinal Flow, Decomposition, and Optical Flow}

Over the past 40 years, many theories have been offered about how people use visual information to find their way. Here, we focus on two theories: differential motion parallax (DMP), information in the retinal flow field; and the structure of information in the optical flow field (IOF). ${ }^{6}$ (See Andersen, 1990; Cutting, 1986; Koenderink, 1986, 1990; Warren, Morris, \& Kalish, 1988; and W. H. Warren \& Hannon (1990) for discussion of other theories; and Owen, 1990, for discussion of terms associated with other theories.) We defer systematic discussion of the IOF until after we present a full logical and empirical analysis of DMP. Before discussing either, however, we must contrast the two theories according to the major way in which they differ.

Again, retinal flow is the field of motions projected on the retina relative to the fovea (where an individual is looking); optical flow consists of these motions projected onto a spherical surface around a moving observer, where that surface is anchored to the coordinates of the environment. With respect to wayfinding, most contemporary approaches start with retinal flow and, through consideration of mathematics and logic, decompose that swirling vector field into at least two component fields. Thus,

$$
\text { Retinal flow } \Leftrightarrow \text { rotational flow }+ \text { optical flow. }
$$

The first component, rotational flow, is due to eye movements; the second is due to observer translation. ${ }^{7}$ As most earlier researchers have called this second component optical flow (e.g., Clocksin, 1980; J. J. Gibson, 1966; Johnston, White, \& Cumming, 1973; Nakayama \& Loomis, 1974), we follow suit. Notice in Equation 4 that when there is no eye movement, retinal and optical flow fields are identical; this fact is important for our final discussion.

Care must be used with the term optical flow. It is used generally for flow patterns of several possible types (e.g., Cutting, 1986; Lee, 1980; Prazdny, 1981, 1983; Radford,
1986; Warren et al., 1988)-such as linear translation and curvilinear translation - and with the possibility of additional components. Thus, we use the term in connection with all exteroceptive (observer) movements. That is, any time the eye is displaced in three-dimensional space (3D), laterally, vertically, or in depth, that motion generates optical flow. In "pure" optical flow (for a linear movement path), there is a point from which all vectors radiate. The locus of this point has often been thought to be used as the aimpoint for wayfinding (Calvert, 1950; J. J. Gibson, 1950, 1966; J. J. Gibson, Olum, \& Rosenblatt, 1955).

Consider the sample flow patterns-retinal, rotational, and optical-shown in the top three panels of Figure 2. Because rotational and optical components add to produce retinal flow, reading Equation 4 from right to left, it has been thought the equation might also be reversed, that is, from left to right. This process is called decomposition, but we present four preliminary arguments against its plausibility.

First and logically, reversing how one reads the equation may seem problematic: For example, consider $4 \Leftrightarrow 3+1$. Although it is unequivocal that 3 plus 1 equals 4 , it is not always the case that 4 should be decomposed into 3 plus 1 . In fact, induction tells us there are an indefinitely large number of possibilities. ${ }^{8}$

\footnotetext{
${ }^{6}$ We avoid the use of the term focus of expansion, a singularity in the optical flow pattern espoused by J. J. Gibson (1966) as the source of information for wayfinding. First, as noted by Warren, Morris, and Kalish (1988), it is technically a misnomer, as the rate of expansion at the focus is zero. Second, as shown by Regan and Beverley (1982; see also Cutting, 1986), other singularities can occur in the optical and retinal flow fields as well. If an observer moves forward and fixates an object slightly off to one side, a singularity indicates where the observer is looking, not where he or she is going. In response to Regan and Beverley, a colloquy followed among Priest and Cutting (1985), Torrey (1985), and Regan (1985). Torrey and Regan agreed that Regan and Beverley's analysis was about a retinal singularity, not an optical one, but Priest and Cutting disagreed. In fact, Cutting (1986) suggested that the optical singularity rarely exists except under very special circumstances. In particular, it cannot occur for observers traversing curvilinear paths.

${ }^{7}$ Rotational flow has also been called propriospecific flow, axial flow (Longuet-Higgins \& Prazdny, 1980), and solenoidal flow (Koenderink \& van Doorn, 1981; Koenderink, 1986). Optical flow has also been called exterospecific flow, polar flow (Longuet-Higgins \& Prazdny, 1980), and lamellar flow (Koenderink \& van Doorn, 1981).

${ }^{8}$ The computational retort to this issue is based on two facts. First, in the retinal flow field, vector lengths corresponding to any object at any particular latitude (measured against the axis of rotation) are equal regardless of their location in space. Second, many equatorial vector lengths in optical flow are very nearly zero (corresponding to points on the horizon). Thus, decomposition is mathematically tractable if, during horizontal eye movements, one can find a patch of relatively small retinal field vectors. These should correspond to a reasonably distant horizon. If one uses the direction of these vectors to determine the amount of rotation and the axis of the rotational field, then subtracts the rotation field from the retinal flow field, the optical flow field will generally result. However, even with such a scheme, one must always settle for approximations (Koenderink \& van Doorn, 1987; Nakayama, 1985).
} 


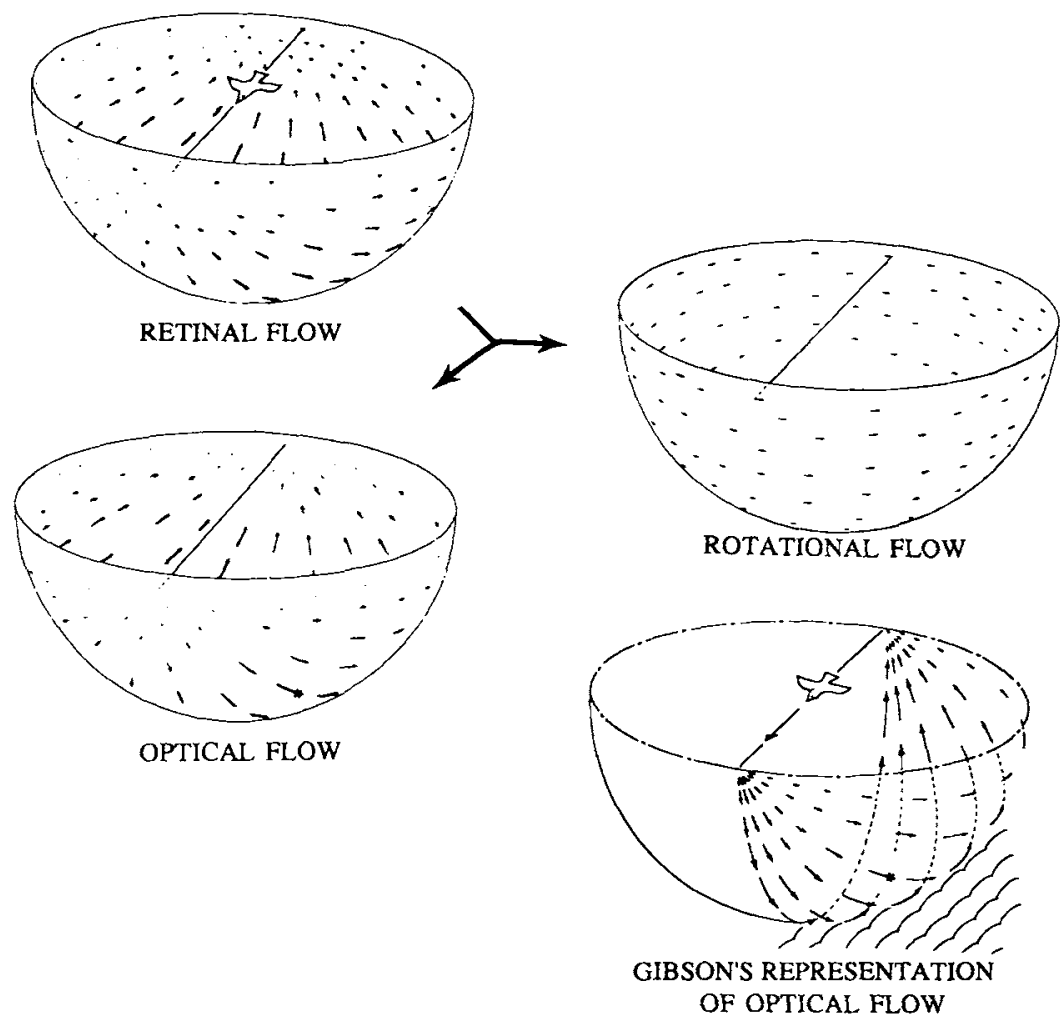

Figure 2. Four hemispherical vector fields of flow. (Top left panel: Retinal flow for bird flying in straight line and looking off to its right side. Top-right and lower-left panels: Decomposition of field into rotational flow [due to eye movements] and optical flow [due to forward movement]. Lower right panel from The Ecological Approach to Visual Perception, p. 123, by J. J. Gibson, 1979, Boston: Houghton Mifflin. Copyright 1979 by Eleanor J. Gibson. Reprinted by permission. Vector lengths around aimpoint are overestimated in Gibson's figure. To make the bottom two panels comparable, vectors marked with a dot at their head were equalized, and all other vectors in the computed field normalized to it. To generate a vector this length the bird had to move forward 0.50 eye heights over the plane. Rotational field indicates $2^{\circ}$ clockwise rotation of eye or head.)

Second, decomposition defies parsimony. On the basis of the data to be presented here, we think information for wayfinding is directly available in retinal flow. If the computational process of decomposition is not needed, why postulate it?

Third, there is empirical evidence against decomposition by the human visual system. Simpson (1988) measured discrimination thresholds for judgments of time to contact (Lee, 1980 ) with an object in the environment in two conditions, one with only optical flow and one with optical plus rotational flow. Thresholds for optical plus rotational flow were elevated over conditions involving only optical flow. Such a result falsifies a decomposition scheme proposed by Koenderink (1985) that is based on looming detectors whose job it would be to filter out rotational motions. More broadly, Simpson's results raise the question of whether people perform decomposition at all.

Finally, a comparison of the optical flow pattern as drawn and presented by J. J. Gibson (1966) with the computed optical flow pattern in the lower panels of Figure 2 reveals two things. First, by convention, the flow patterns represent what a bird might see flying over a flat, uncluttered terrain. Clutter removes the spatial regularities seen in the flow pattern. Second, and more important, the vectors in Gibson's array (see also Turvey, 1990, p. 942) are overly optimistic in the direction of movement. In the computed flow pattern, the vectors are very small near the aimpoint, and there is very little structure in IOF within a circular region of about $10^{\circ}$ if not larger. If an individual did perform decomposition, he or she would have to extrapolate over relatively large regions of the visual field to find the aimpoint.

Despite the apparent force of these four arguments, however, Warren et al. (1988) presented evidence in support of decomposition and in support of the use of IOF. We return to their data, but first we present our nondecompositional approach to wayfinding.

\section{Retinal Flow and Differential Motion Parallax}

What is DMP? Cutting (1986) proposed that mobile observers use the relative motion and velocities of near and far objects against a fixated object to determine their aimpoint. 
That is, given an object to fixate and to pursue, in most environments near objects will generally move faster than, and in the opposite direction from, far objects. A mobile observer need only shift his or her gaze opposite to the most rapid flow in the retinal array to find the eventual instantaneous aimpoint. ${ }^{9}$ Here, we start with a different representation of optical flow (see also Cutting, 1986), then modify it for retinal flow.

\section{Optical Flow as a $3 D$ Vector Field}

Let $x, y$, and $z$ be Cartesian coordinates for any object around an observer ( $z$ indicating depth behind the picture plane, $x y$ ), where the observer's eye is at the origin of the system. ${ }^{10}$ From these values, one can then convert to polar coordinates with unit radii:

$$
\begin{aligned}
\theta & =\arctan (x / z), \quad \text { and } \\
\phi & =\arctan \left[y /\left(x^{2}+z^{2}\right) \chi\right],
\end{aligned}
$$

where $\theta$ is the angle to a given object in the horizontal $(x z)$ plane in a great circle around the observer's eye, and $\phi$ is the vertical angle to same object above or below that plane. When the observer moves forward along the $z$-axis, these formulae are differentiated to achieve the flow of all objects in an environment represented in polar coordinate vectors. The new formulae are

$$
\begin{gathered}
\mathrm{d} \theta / \mathrm{d} z=-x /\left(x^{2}+z^{2}\right), \quad \text { and } \\
\mathrm{d} \phi / \mathrm{d} z=-y z /\left[\left(x^{2}+y^{2}+z^{2}\right) \operatorname{sqrt}\left(x^{2}+z^{2}\right)\right] .
\end{gathered}
$$

Notice that when $x=0$, Equation 8 simplifies to

$$
\mathrm{d} \phi / \mathrm{d} z=-y /\left(y^{2}+z^{2}\right)
$$

similar in form to Equation 7.

Analytically, the horizontal $(x z)$ plane is a nested set of figure eights, all with inner tangents at the origin, as shown in the upper right panel of Figure 3. Given Equation 9, the vertical $(y z)$ plane is identical to the horizontal. Moreover, all planar slices through the $z$-axis have the same form. Thus, when rotated around the $z$-axis, the 3D form of the vector field is a nested set of toroids; one is suggested in the upper middle panel of Figure 3. Similar analyses are given by Gordon (1965), by Whiteside and Samuel (1970), ${ }^{11}$ and by Koenderink (1985), who, generalizing from horopter analyses, called the structure in the upper middle panel of Figure 3 a Vieth-Müller torus.

These nested figure eights in a planar field and toroids in volumetric field represent the instantaneous isoangulardisplacement contours in the world for an observer who happens to be moving in a straight line and looking at his or her aimpoint. That is, everything on a particular figure eight, or bagel-shaped surface, around the observer's eye moves away from the aimpoint at equal absolute velocity (i.e., ignoring direction). Thus far, we have not deviated from the work of Calvert (1950, 1954), J. J. Gibson (1947, 1950), or Warren et al. (1988); we have simply presented a different representation of the optical flow field, which can be generalized to a space filled with clutter.

\section{Retinal Flow Projected out to Its $3 D$ Vector Field}

This analysis is not yet useful. Because an observer looking in his or her direction of linear locomotion is the potential and hoped-for end result of an aimpoint-finding process, it cannot also be its beginning. To start at the beginning, following von Kries (Helmholtz, 1925, pp. 371-372), we need to consider the mobile observer with a mobile eye, looking at and pursuing any object in the environment. Thus, we must consider $3 \mathrm{D}$ spatial coordinates corresponding to the retinal flow field.

In wayfinding by DMP, one fixates and pursues an object, holding it in position at the fovea. Thus, it has null retinal velocity. Mathematically, then, one subtracts the particular instantaneous velocity of this object from the entire 3D optical vector field. ${ }^{12}$ Some resulting sets of retinal flow fields are shown in the lower left and lower middle panels of Figure 3. Notice that the new figure eights and new toroid around the moving observer are now deformed and asymmetric. Tighter

\footnotetext{
${ }^{9}$ We emphasize that gaze is shifted against the physically most rapid motion, not the perceptually most rapid motion. Thus, we are not considering the yoking of perceived motions with apparent depth (e.g., Gogel, 1982), but simply the registered motions early in visual process.

${ }^{10}$ Here, and throughout the article, we assume that the observer is functionally monocular. It is unclear what effect, if any, is gained by binocular considerations of differential motion parallax, but see $\mathrm{Re}$ gan and Beverley (1978; Regan, Kaufman, \& Lincoln, 1986) for a psychophysical analysis of binocular information about looming objects. In addition, Roy and Wurst (1990) found that certain neurons in the medial-superior-temporal area of the macaque responded to differential motion according to disparity, crossed versus uncrossed. In particular, given a particular fixation, neurons responded to the motion of nearby objects (cross disparities) in one direction and far objects in the other (uncrossed disparities). Unfortunately, as the fixation distance of their macaques was less than $1 \mathrm{~m}$, the uncrossed response of these neurons could not be used for wayfinding, as neither macaques nor humans look nearby when they want to know where they are going. Moreover, the inward motion (IM) analysis in Experiments 1 and 2 shows that nearby objects (with crossed disparities) are not needed for wayfinding. We suggest Roy and Wurst found "rocking-chair" neurons, those active, for example, when one is rocking in a chair in one's office while talking to a colleague.

"Gordon (1965) and Whiteside and Samuel (1970) based their analyses on temporal derivatives rather than spatial derivatives because their interest was in velocities of objects as blurred by highspeed landing approaches of aircraft. The patterns of temporal and spatial derivatives, however, are identical in shape because forward movement in space or in time causes the observer to have the same spatial relationships with surrounding stationary objects.

${ }^{12}$ As noted in Footnote 8 , the vector subtraction process is a bit more complicated than this. If the plane going through the aimpoint, the eye, and the fixated object is considered the equator of the optical field, then the vectors subtracted from latitudes higher and lower in that field are smaller (corresponding to the cosine of the declination times the original vector length). Thus, at the poles of the axis of eye rotation, for example, no vector is subtracted. Notice also that vector subtraction (due to preservation of vector direction) will add to motion (make it faster) on one side of gaze direction and subtract from it (make it slower) on the other.
} 

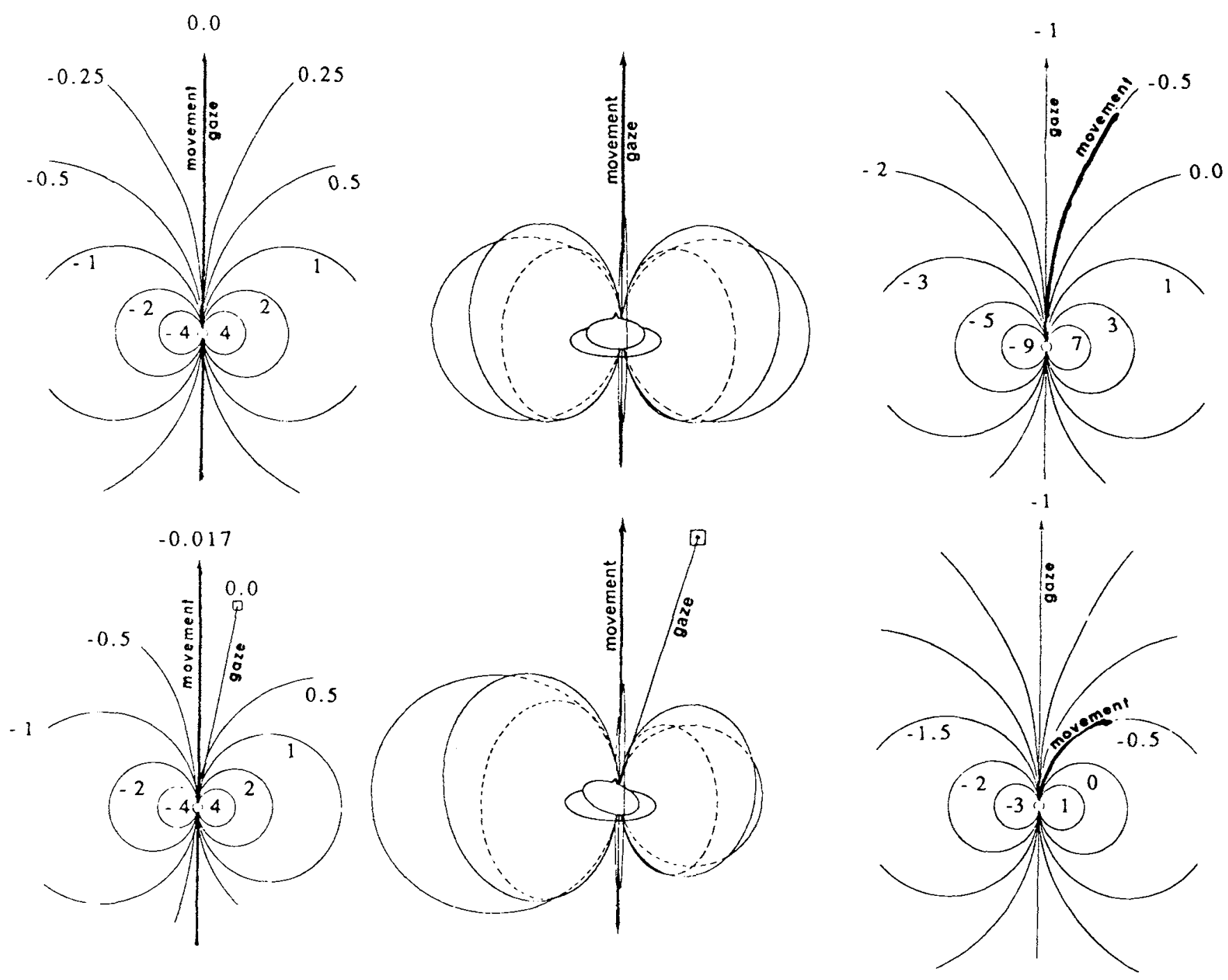

Figure 3. Isoangular-displacement contours and surfaces for differential motion parallax (DMP). (Top left panel, isoangular-displacement contours in horizontal plane through eye of observer moving along straight path and looking in direction of motion; bottom-left panel, contours for individual looking at object to the right side; top-left panel, different representation of optical flow field [retinal field without rotations]; bottom-left field is a different representation of retinal flow field. Middle panels are representations of three-dimensional instantaneous isoangular-displacement surface for linear locomotion with gaze fixed along path [top-middle panel] and gaze at object off to the right [bottom-middle panel]. Right panels are representations of isoangular-displacement contours for two curved paths. Notice the similarity to the right panels demonstrates that DMP applies to curvilinear and linear paths equally.)

contours (and hence more rapid velocities) occur on the fixation side of the movement path.

\section{Rules for Wayfinding}

Most pertinent to this analysis, and going beyond previous work (Gordon, 1965; Koenderink, 1985, 1990; Koenderink \& van Doorn, 1987; Whiteside \& Samuel, 1970), is a fact about a set of three objects, all roughly along the same line of sight and not along the path of movement. Consider the following: Object 2 is an object under scrutiny (foveated on the retina), Object 1 is any near object, and Object 3 is any farther one. For a scrutinized object at any location (Object 2), a second object half the distance to the first (Object 1) will move faster than, and in the opposite direction from, all possible objects (Objects 3 ) farther away than Object 2 . This inequality is invariant regardless of where one looks, fore or aft, up or down, or near or far. It can be expressed simply as

$$
N>-F \text {, }
$$

where $N$ is the retinal velocity of any near Object 1 at most half the distance to Object 2 (and given positive sign) and $F$ 
Table 2

Rules for Wayfinding by Retinal Flow

\begin{tabular}{|c|c|}
\hline Rule & Description \\
\hline Step 1 & $\begin{array}{l}\text { While moving forward, fixate and pursue (if necessary) any } \\
\text { object of potential interest in your environment. While } \\
\text { doing so, register any motion across the plane of sight. }\end{array}$ \\
\hline Condition 1 & $\begin{array}{l}\text { If there is no motion across the plane of sight, you are } \\
\text { likely to be looking in the direction you are going. If } \\
\text { there is such motion, go to Step } 2 \text {. }\end{array}$ \\
\hline Step 2 & $\begin{array}{l}\text { Register the directions and velocities of objects around the } \\
\text { fixated and pursued object (as they project on the } \\
\text { retina). Your aimpoint is likely to be in the direction } \\
\text { opposite the most rapid retinal flow [by an amount } \\
\text { proportional to its value]. Is the current motion } \\
\text { information adequate as an update of your current } \\
\text { aimpoint? }\end{array}$ \\
\hline Condition 2 & $\begin{array}{l}\text { If the motion information is adequate, continue with Step } \\
2 \text { (or, if you choose, go back to Step } 1 \text { for a new object). } \\
\text { If the motion information is inadequate, saccade in the } \\
\text { direction opposite from the most rapid motion [by an } \\
\text { amount proportional to its value] and go back to Step } 1 \text {, } \\
\text { picking a new object. }\end{array}$ \\
\hline
\end{tabular}

The plane of sight the vertical, or $y z$, plane through both the eye and the object under fixation.

is the retinal velocity for any possible far Object 3 . Thus, all a moving observer need do to determine his or her aimpoint is to follow a recursive set of rules, given in Table 2.

What is particularly pleasing about this set of rules is that they work whether the observer is moving along a straight path or a curved one. The vector fields for two curved paths
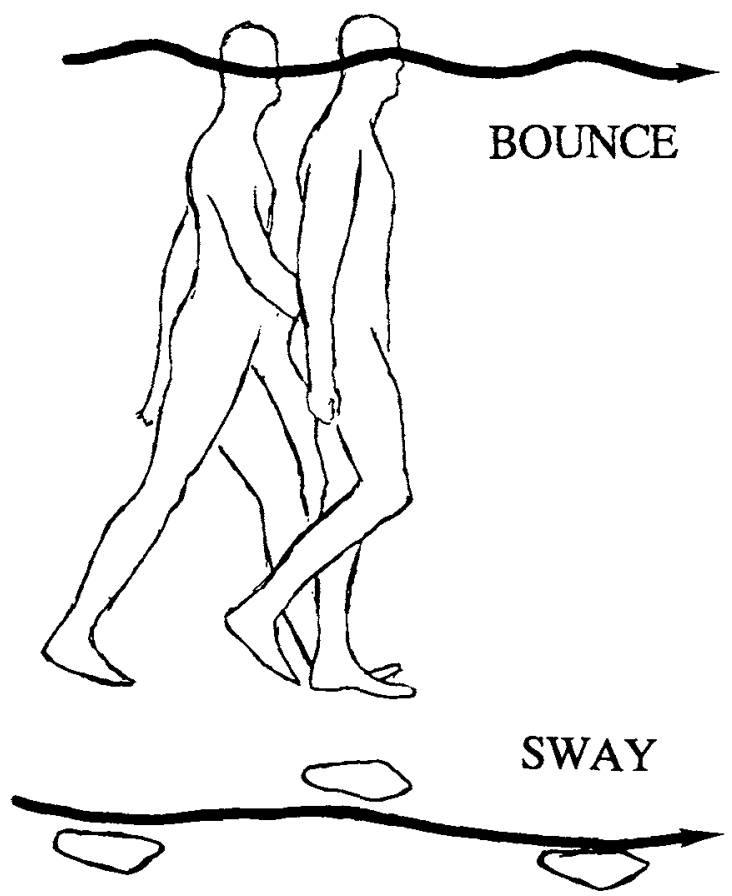

Figure 4. The vertical (bounce) and horizontal (sway) components of gait. (During pursuit fixation each movement will add two components to retinal flow, one due to counterrotation of the eye during pursuit fixation and one due to displacement of eye position in threedimensional space.) are shown in the right panels of Figure 3; the derivation is given in Cutting (1986, pp. 209 and 285). Notice that the shape of these nested figure eights is identical to those in the left panels for a linear path; the scale of relative velocities is shifted, but if the observer nulls the flow of any point in this vector field by gazing at an object, he or she creates the same instantaneous invariant inequality in the entire field as given in Equation 10. Moreover, the same recursive steps given in Table 2 would prove useful, with one exception: Because the instantaneous direction of movement is constantly changing, a mobile observer never achieves a positive answer to Conditional 1. Note two additional aspects of the right panels of Figure 3.

First, the null velocity points are on a curved line bending in the direction of locomotion. Raviv (1990) has used this line of zero curvature for purposes of the study of camera fixation in robotics. This curve is problematic for any notion of an aimpoint as a singularity in the optical flow field, because null velocity points change position at each distance along the curved path. Obviously, this is no longer a straight line seen end on, and hence a point. ${ }^{13}$ Moreover, these null velocity points are not even on the circular path of movement, and no focus of expansion can exist.

\section{Bounce, Sway, and More on Decomposition}

Second, and more pertinent to this article, curving paths are integral to all natural forms of human locomotion. As one walks or runs through any environment one not only advances through space but also generates a vertical and hori-

\footnotetext{
${ }^{13}$ Molyneux (1690) posed the famous argument against perception of objects in depth; essentially, the $z$-axis is a line seen end on and hence is a point. Berkeley $1703 / 1937$, picked up on this idea, and ironically, even J. J. Gibson, Olum, and Rosenblatt (1955, p. 373) and E. J. Gibson, Gibson, Smith, and Flock (1959) used it against the use of motion parallax information for depth and wayfinding.
} 
zontal oscillatory movement, as suggested in Figure 4. That is, even an observer's best effort at maintaining a perfectly straight dollying-like path is confounded by footfalls and leg extensions that necessarily alter the path of the head and eye, up and down and side to side (see Carlsöö, 1972; Cutting, Proffitt, \& Kozlowski, 1978; Murray, 1967). We call these oscillations bounce and sway; sway is generally greater for a walker than for a runner, but bounce and sway occur for both. Moreover, these are conditions that visual systems have had to contend with since locomotion on land began.

Murray (1967) found that bounce is well approximated by sinusoidal oscillation in eye height of $6.0( \pm 1.3) \mathrm{cm}$ at a rate of twice per step cycle, and sway by lateral sinusoidal oscillation of about $5.8( \pm 2.0) \mathrm{cm}$ once per cycle. As her individuals averaged $1.73 \mathrm{~m}$ in height, the range within \pm 2 standard deviations for both motions is from $1.0 \%$ to $5.7 \%$ of eye height. The consequence of these movements is that if the observer fixates a particular object in a particular location, all other objects in the retinal array around it will move (bouncing and swaying) relative to the array. Casual observation and reflection during a stroll outdoors will confirm this set of additional motions in retinal flow.

Bounce and sway during locomotion with a fixed gaze add four additional components to retinal flow. That is, each oscillation adds one sinusoidal component due to translation of the eye position (optical flow) and one compensatory counterrotation of the eye to maintain fixation (rotational flow). The repetitiveness and rhythmicity of gait make the small counterrotational eye movements predictable and accurately executed.

Let us assume that an observer fixates an object off to the side at an elevation of one eye height during gait. Equation 4 thus becomes complicated and has the form

Retinal flow $\Leftrightarrow$ rotational flow for horizontal pursuit fixation (around $y$-axis) +

rotational flow for bounce (around $x$-axis because of oscillating counterrotation of the eye) +

rotational flow for sway (around $y$-axis because of oscillating counterrotation of eye) +

optical flow for bounce ( $y$-axis oscillating translation in eye position) +

optical flow for sway ( $x$-axis oscillating translation in eye position) +

optical flow for linear translation.

Notice that any decomposition scheme, reading Equation 11 from left to right, must make assumptions about the number, extent, and linkage of flow components. During a stroll along a straight path, five flow fields must be subtracted from retinal flow before the radial pattern of the linear translational component (optical flow) could reveal the aimpoint. Such computation begins to look complex. ${ }^{14}$

However, no extra assumptions or computations are necessary with DMP. The four new components are irrelevant to the inequality in Equation 10, as DMP is equally operable under linear and curvilinear movements, so long as gaze is fixed. One need only integrate over time to obtain the average heading. Thus, the addition of bounce and sway components to simulated locomotion, at least in theory, ought not impede wayfinding performance; DMP should work equally for a pedestrian and for a passenger on a train.

\section{DMP Displays Use a Simulated Pursuit Fixation Technique}

Before discussing evidence for DMP, we must discuss the structure of the stimulus display necessary to present it, that is, simulated pursuit fixation. It is identical to a combined dolly and pan shot in television or film (see Hochberg, 1986; Macgowan, 1965). It is also a fine way to make a rigid environment appear interesting; as Spottiswoode (1968) noted, "when the world of reality has been frozen, the movement of the camera can bring it back to life" (p. 122).

Imagine a camera on a jeep moving through a savannah, looking off to the right side at an animal. As the jeep drives forward, the camera pans right to keep the animal in the middle of the field of view. The movements of foreground textures and objects against fixation are more rapid than and are opposite to the background textures and objects; this pattern of motion is DMP. It is not completely clear why simulated pursuit fixation works psychologically, but it does and effects important in nearly a century of cinema are based on it. ${ }^{15}$ The efficacy of simulated pursuit fixation must be based on an observer's unconcern with the broken linkage between global retinal motions and the eye movement efference and afference associated with them.

With simulated pursuit fixation displays, the experimental variable of interest is the angle between lines of gaze and movement, called the gaze-movement angle (Cutting, 1986). Typically, as one approaches a fixated object that is off the path of movement, the gaze-movement angle grows. The maximum value of this angle during the trial is the independent variable of interest. The maximum, rather than the minimum or mean, gaze-movement angle is chosen to be appropriately conservative; all DMP information within a trial is based on this angle or less. The shape of the performance function across different, final gaze-movement angles can be used to determine the empirical accuracy of wayfinding.

Neurophysiological analyses of relative motion for other creatures suggest that visual systems could pick up DMP

\footnotetext{
${ }^{14}$ At any one instant, retinal flow can be decomposed into one optical flow field and one rotational flow field. However, the task of wayfinding is not done in an instant, and thus the complications of the other flow fields quickly enter. Given that stimuli of 3-s duration seem to be needed for wayfinding (as shown by the results of Experiment 3 ), the other optical and rotational fields clearly manifest themselves.

${ }^{15}$ It is not known when the first dolly and pan shots were incorporated into film. Camera movement began as early as 1896 , but only as a result of mounting cameras on a boat, trolley, or even a gondola. By 1912, D. W. Griffith also mounted a camera on a car. Among popular and successful films, the earliest pan may be in Edwin Porter's The Great Train Robbery, which appeared in 1903. Combined dolly and pan shots have existed at least since 1915 (Macgowan, 1965).
} 
(Allman, Miezin, \& McGuiness, 1985; Bridgeman, 1972; Frost \& Nakayama, 1983; Judge, 1990; Nakayama, 1985; Roy \& Wurst, 1990), and machine vision studies have implemented DMP-like schemes (Rieger \& Lawton, 1985). But does the human visual system use DMP?

\section{Previous Evidence From Simulated Pursuit Fixation Displays}

Four groups of studies have been performed using simulated fixation techniques. First, but least consequential, Regan and Beverley (1982), using sinusoidal gratings, found that observers could not determine their aimpoint within $20^{\circ}$ gazemovement angle. However, their results are not surprising as their displays simulated near-orthogonal approach to a single, nonrigid plane (see Priest \& Cutting, 1985). Researchers usually assume both rigidity and depth are necessary for wayfinding from either optical or retinal flow (see our Experiment 9).

Second, Rieger and Toet (1985) simulated observer rotations and translations toward two transparent parallel planes at different depths, each speckled with random dots. With well practiced observers, Rieger and Toet found about $85 \%$ performance in a four-choice task for gaze-movement angle of $5^{\circ}$, but only about $60 \%$ performance for angles of $2.5^{\circ}$. Adjusting these values to a two-choice task would yield about $93 \%$ and $80 \%$, respectively. As in the Regan and Beverley (1982) study, performance degenerated to chance when there was no depth difference between the planes. The two-plane performance seems adequate for wayfinding tasks, but Rieger and Lawton (1885) presented no object or texture at their computed fixation point. Rieger and Toet (1985; see also Rieger \& Lawton, 1985) also couched their findings in terms of decomposition, subtracting a field of difference lines from retinal flow to obtain optical (translational) flow. ${ }^{16}$ We see no need to go beyond analysis of the retinal information.

Third, Cutting (1986; see also Cutting, 1983) simulated a sparse environment consisting of four vertical posts (lines, with tops and bottoms not visible) randomly positioned on each of three invisible, parallel planes. For linear translations and with greatest depth differences between planes, performance was above chance $(33 \%)$ at a final gaze-movement angles of $0.5^{\circ}$ and was above $80 \%$ at angles of $5^{\circ}$ and more. In two other experiments curved paths were simulated with similar, even superior, results. Unfortunately, there are three problems, as noted by Warren et al. (1988) and Warren and Hannon (1990): (a) Feedback was given after each trial; and thus the findings might represent learning a nonsense visual task unrelated to wayfinding; (b) each stimulus was presented three times before observers responded; and (c) as with Rieger and Toet (1985), no object was provided at the simulated fixation point.

Fourth, Warren and Hannon (1990) simulated observer movement across a speckled plane, with and without simulated fixations on an object in the foreground. They found no difference between the two conditions and $75 \%$ accuracy at less than $2^{\circ}$ gaze-movement angle. Like Rieger and Toet (1985), Warren (Warren et al., 1988; Warren \& Hannon, 1990) also embraced a decompositional approach, preferring to refer these results to the use of IOF. We discuss some of Warren's results in detail later.

For our purposes, all of these studies share the same problem: None of them simulated the bounce and sway motions generated by a bipedal, moving observer. Thus, one cannot yet generalize the DMP wayfinding technique to bipedal locomotion and explore the complexities of Equation 11.

\section{Experiment 1: DMP, Gait, and Obstacle Avoidance}

We had two goals in the first experiment: to add the bounce and sway of natural gait to the retinal motions in the stimuli and observe their effects. if any; and to rectify the problems inherent in the studies of Cutting (1986) by presenting stimuli with an object at simulated fixation once (as opposed to three times) and without feedback.

\section{Method}

Stimuli. Stimuli were precomputed and stored as disk files on a Hewlett-Packard (HP) 1000L Series computer. These were fetched during the experiment and displayed on an HP 1350 S vector-plotting display with a $1,024 \times 1,024$ point-plotting resolution. Each stimulus contained seven schematic, small "trees," one at simulated fixation (in the center of the screen) and six whose relative motions varied over the course of the trial. The $x$ - and $z$-coordinates of the six nonfixated trees were randomly generated with the constraint that one fall in each of the rectangular sections shown in the right panel of Figure 5. Each tree was a phosphor green cross on a black background with no horizon visible. The horizontal crossbar was at the observer's eye height and the vertical trunk was two eye heights in length, as suggested in the left panels of Figure 5. DMP is most pertinent to the near and far trees.

Motion was generated across 55 frames, each presented for 107 ms, for a total trial duration of about $5.9 \mathrm{~s}$. Stimuli were presented once. The fixated tree was at a distance of 13.7 eye heights (about 22 $\mathrm{m}$ for an individual with an eye height of $1.6 \mathrm{~m}$ ), and the simulated distance traveled was 6.85 eye heights. Simulated forward velocity was 1.16 eye heights $/ \mathrm{s}$ (or about $1.85 \mathrm{~m} / \mathrm{s}$ ), a value that is just below the lowest velocity considered in Table 1 . This places the experimental situation within the distance range considered in Figure 1 and the velocity range of Table 1 , thus making the task one of obstacle avoidance.

During the course of a stimulus trial (as shown in Figure 5), the cluster of trees enlarged (lines grew longer, but not wider) and filled the screen, creating an image of about $9^{\circ} \times 9^{\circ}$. Stimuli were polarprojected, and viewers sat near the projection point at a distance of about $1 \mathrm{~m}$. From there, the resolution of the display was 100 address-

\footnotetext{
${ }^{16}$ Wayfinding by DMP and wayfinding by Rieger and Lawton's (1985) differential motion (DM) are quite similar, but at least two points separate them. First, DM has more logical steps. In DM (a) the difference lines in retinal flow are computed for points within a neighborhood by minimizing the sum of squared perpendicular distances among them; then (b) these lines are subtracted from the retinal flow, and a good approximation of optical flow is obtained with its aimpoint visible. According to DMP, however, the second step is not needed; the magnitude of the difference vectors is the information. Second, DMP can fail (e.g., with objects nearer than fixation not sufficiently close to the observer to counter the opposing motions of far distance object), but the DM difference lines may still point in the direction of the aimpoint.
} 

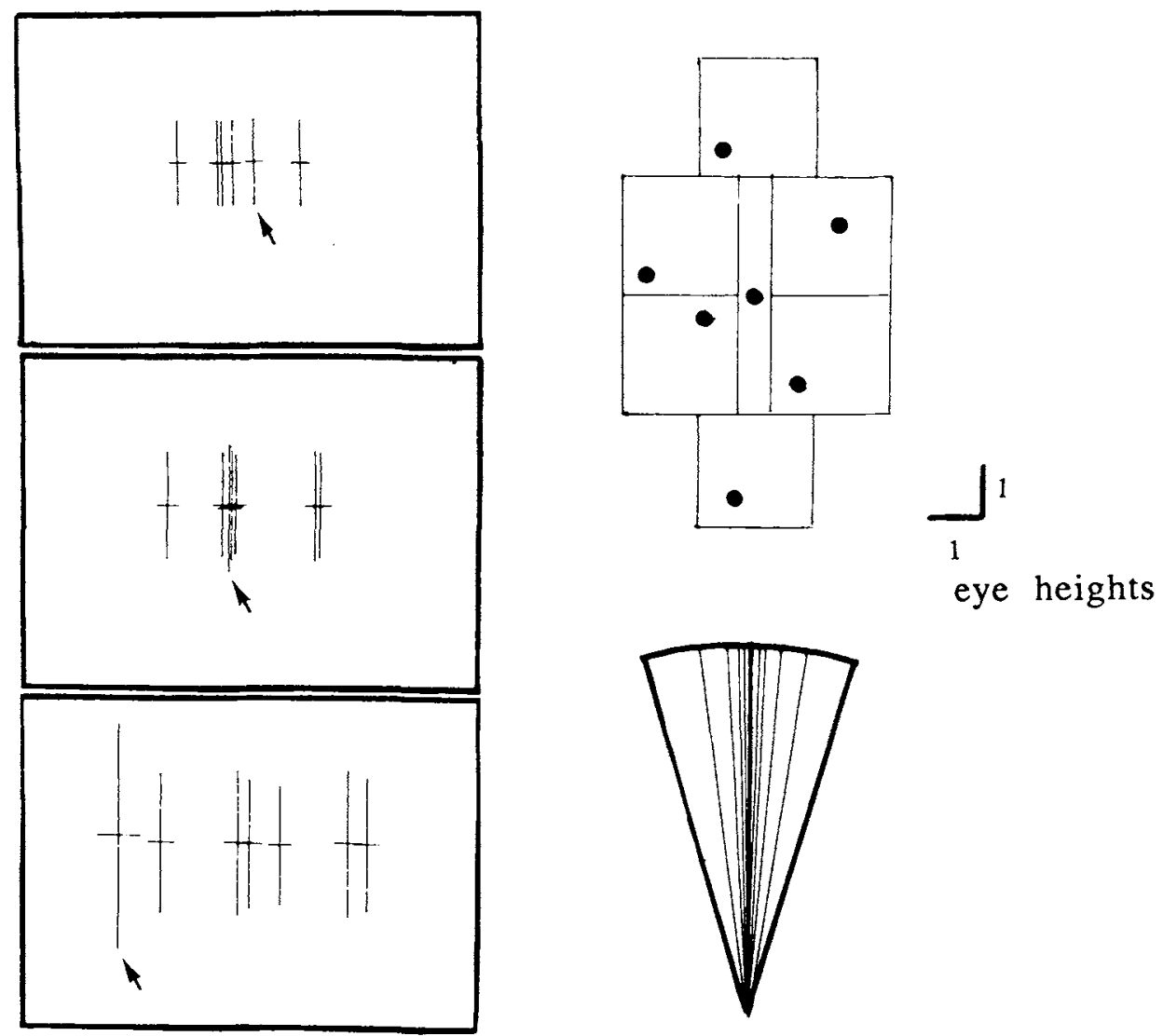

Figure 5. Stimulus frames and the environments for Experiment 1 . (In the left panels are three sample frames $[1,23$, and 45] taken out of a 55-frame stimulus sequence in Experiment 1 with bounce and sway components of observer movement. The fixation trees are in the middle of each frame, and the tree indicated with small arrows at its base is the tree generating differential motion parallax; it moves faster than and opposite to all more distant trees. Thus, these panels represent a trial with observer movement to the right. In the right panel is a bird's-eye view of the environment and the paths taken through it. One tree [at the center] appeared at fixation, and six others were stochastically planted around it within fixed rectangular regions. The fan at the bottom suggests the scaled lengths and angles of paths taken in the experiment, to the left and right of the fixation tree.)

able locations per degree of visual angle. Viewing was unconstrained and binocular and took place in a moderately lit room. Edges of the display, for example, could clearly be seen.

Half the stimuli were generated by simulating a smooth dollying observer movement with a pan to the side, as in Cutting (1986), and half were generated with additional bounce and sway calibrated to natural gait and pursuit fixation. Bouncing movements were $5.6 \%$ of simulated eye height and swaying movements were $4.8 \%$, both within the upper ranges found for natural gait by Murray (1967). A bounce and sway trial sequence simulated 12.5 steps, or 6.25 step cycles. All such trials started as if on the right foot. Half of the trials presented simulated observer movement to the right of the fixated tree, and half of the trials presented simulated movement to the left. Coordinate positions of the seven trees were identical in matched quadruples of stimuli, with and without bounce and sway and with simulated fixation to the left and right of the path of movement.

Fifty-six stimuli were randomly intermixed and presented in two different sequences to each participant, yielding 112 trials. The set of stimuli included 7 final gaze-movement angles $(0.25,0.5,1,2,4,8$, and 16) $\times 2$ gaze directions (left and right of the path of movement) $\times 2$ carriage conditions (with and without bounce and sway) $\times 2$ tokens (with different stochastically generated positions of the six nonfoveated trees). In all cases, initial gaze-movement angles were about half the final gaze-movement angles.

Procedure. Eight graduate and undergraduate students at Cornell University participated individually. These participants and others in later experiments had normal or corrected-to-normal vision. The 8 participants in this experiment were unfamiliar with the experimental hypotheses. They were told they would be watching stimuli simulating their own movement through an environment populated with seven schematic trees and that stimulus motion would emulate their fixation on a center tree. They were encouraged to keep their gaze at midscreen, but eye movements were not monitored. Viewers pressed 1 on a keyboard when they thought they were looking left of their path of movement and 0 if they thought they were looking right. We call this a nominal direction task. No reaction times were recorded. Participants were given six practice trials to familiarize them with the stimuli, and then the experimental sequence began. No feedback was 

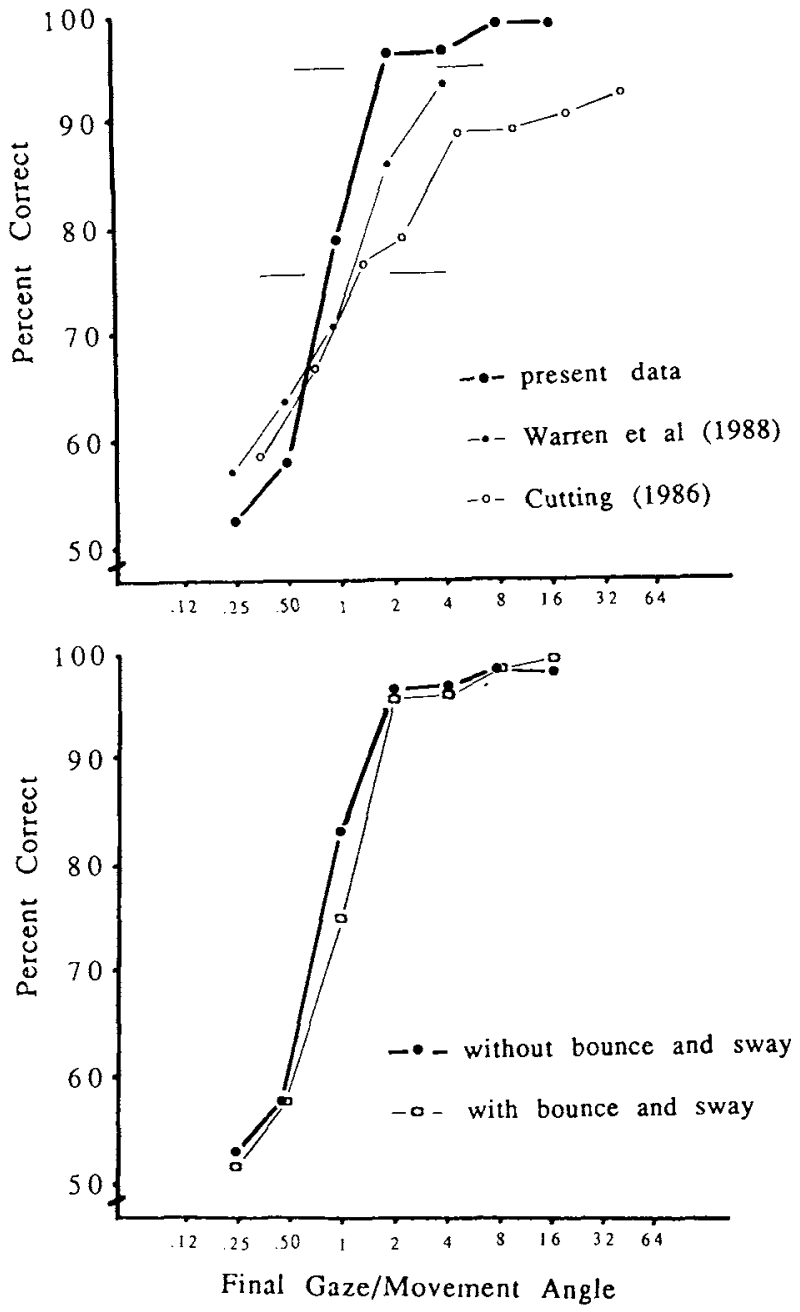

Figure 6. Data from Experiment 1. (Top panel compares observer performance at the final gaze-movement angles in Experiment 1 for differential motion parallax (DMP) in retinal flow with the data of Warren, Morris, \& Kalish [1988, Experiment 2, 63 dots, for information in optical flow and with the data of Cutting [1986, Experiment 9, Condition 1; with its range adjusted for a two-alternative task] for DMP in retinal flow. Superimposed on these functions are the $75 \%$ criterion proposed by Warren et al. and the $95 \%$ criterion proposed here. The bottom panel compares performance on trials with and without bounce and sway.)

given. The experiment lasted about $45 \mathrm{~min}$, and participants were paid $\$ 5$.

\section{Results and Discussion}

The overall pattern of results (shown in Figures 6 and 7) improved on the pattern of Cutting (1986) and even W. H. Warren et al. (1988), as seen particularly in the top panel of Figure 6 . Wayfinding performance here was essentially perfect for final gaze-movement angles of $2^{\circ}$ or more, above chance for angles of $1^{\circ}$ and $0.5^{\circ}$, and near chance for $0.25^{\circ}, F(6,42)$ $=100.3, M S_{\mathrm{e}}=84.0, p<.0001$.
Warren et al. (1988) and Warren and Hannon $(1988,1990)$ suggested that the interpolated value in the data corresponding to the $75 \%$ performance level is a reasonable criterion for assessing performance. Rather than fit the mean function as they did, we rescaled the individual performance functions and assessed their best fit to a range-adjusted logistics curve. The median $75 \%$ performance level across subjects was $0.9^{\circ}$.

$A$ stricter criterion. On reflection, however, a $75 \%$ criterion seems much too loose. A jogger in the open woods might run into every fourth tree near his or her path; at such a threshold, joggers might quickly become extinct. Given the importance of the task of wayfinding and the unforgiving consequences of failure to perform it, a criterion of $95 \%$ seems more apt. The median $95 \%$ threshold interpolated from the individual data was $1.9^{\circ}$, still superior to the $3.7^{\circ}$ required for a velocity of $2 \mathrm{~m} / \mathrm{s}$, shown in Table 1 . These criteria and those for various conditions in later experiments reported are shown in Table 3.

Most pertinent to our arguments against decomposition, however, are the results for judgments of the stimuli with and without bounce and sway. As shown in the bottom panel of Figure 6, performance in these conditions was virtually identical, with bounce and sway stimuli yielding only slightly poorer performance, $F(1,7)=5.65, M S_{\mathrm{e}}=1.78, p<.045$. This statistical difference was due entirely to one stimulus with a final gaze-movement angle of $1^{\circ}$. As shown in Table 3 , median $95 \%$ thresholds, if anything, favored the bounce and sway stimuli. The main thrust of the results, we suggest,

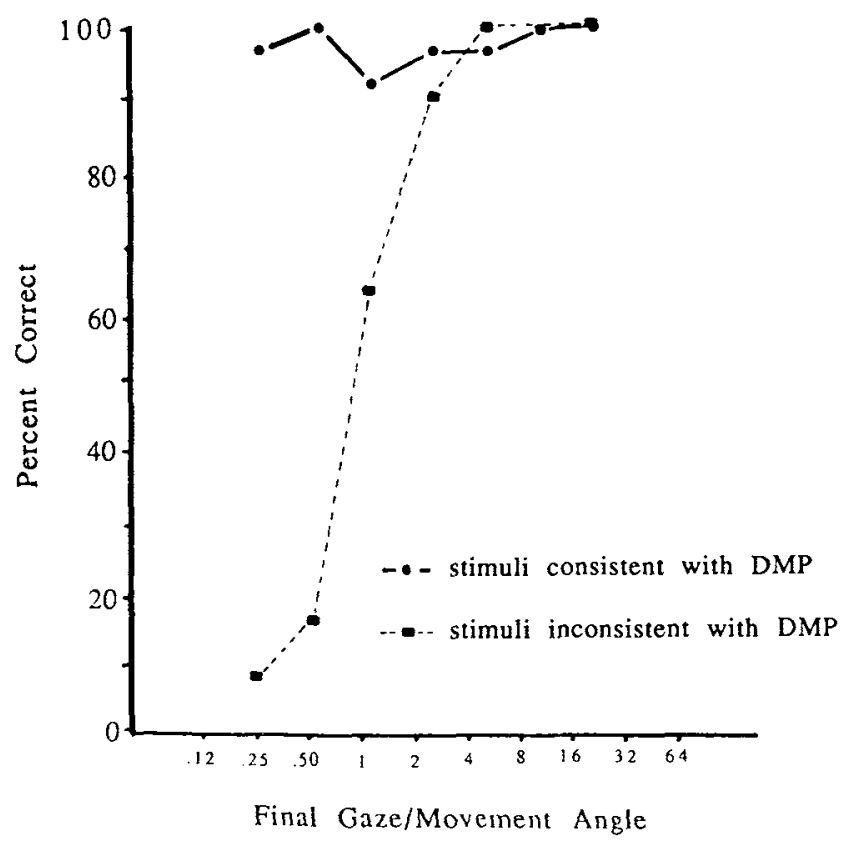

Figure 7. Results of Experiment 1 parsed according to stimuli consistent and inconsistent with differential motion parallax, DMP. (Inconsistency is defined as one or more violations of Equation 10 during the stimulus sequences; that is, at least one farther tree moving faster in the retinal array than and in the opposite direction to all nearer trees. The residual information available beyond $2^{\circ}$ in stimuli inconsistent with DMP is addressed in Experiment 2.) 
Table 3

Median Wayfinding Thresholds for Selected Conditions in the Nine Experiments

\begin{tabular}{|c|c|c|c|c|}
\hline $\begin{array}{l}\text { Displays, information type, and } \\
\text { experiment }\end{array}$ & $\begin{array}{c}\text { Velocity } \\
(\mathrm{m} / \mathrm{s})\end{array}$ & $\begin{array}{c}75 \%^{\mathrm{b}} \\
\text { (degrees) }\end{array}$ & $\begin{array}{c}95 \%^{\mathrm{b}} \\
\text { (degrees) }\end{array}$ & $\begin{array}{l}\text { Subjects meeting } \\
95 \% \text { criterion }^{\mathrm{c}}\end{array}$ \\
\hline \multicolumn{5}{|c|}{ Simulated pursuit fixation and differential motion parallax } \\
\hline Experiment 1: Avoidance, 7 trees & 1.85 & & & \\
\hline Without bounce and sway & & 0.9 & 2.0 & $8 / 8$ \\
\hline With bounce and sway & & 1.0 & 1.5 & $7 / 8$ \\
\hline Experiment 2: Route finding & 2.18 & & & \\
\hline Without bounce and sway & & 1.2 & 5.3 & \\
\hline With bounce and sway & & 1.5 & 6.8 & \\
\hline With 74 trees & & 1.0 & 3.2 & $5 / 8$ \\
\hline With 5 trees & & 1.1 & 8.5 & $2 / 8$ \\
\hline With 74 disks & & 1.6 & 5.2 & $2 / 8$ \\
\hline With 5 disks & & 1.8 & 6.2 & $0 / 8$ \\
\hline Experiment 3: Avoidance, 5 trees & 1.90 & & & \\
\hline Without bounce and sway & & 1.6 & 4.0 & $6 / 12$ \\
\hline With bounce and sway & & 1.1 & 4.2 & $5 / 12$ \\
\hline Experiment 8: Avoidance, 21 trees & 1.90 & & & \\
\hline Without bounce and sway & & 0.6 & 2.4 & $3 / 3$ \\
\hline $\begin{array}{l}\text { With exaggerated bounce and } \\
\text { sway }\end{array}$ & & 1.0 & 3.3 & $3 / 3$ \\
\hline Experiment 9: Avoidance, 21 trees & 1.90 & & & \\
\hline In a rigid environment & & 0.6 & 1.2 & $10 / 12$ \\
\hline In a nonrigid environment & & 1.8 & 18.6 & $1 / 12$ \\
\hline \multicolumn{5}{|c|}{ Fixed camera angle and the information in optical flow } \\
\hline Experiment 4: 74 disks & 2.18 & 1.5 & 6.0 & $2 / 9$ \\
\hline Experiment 5: & 2.18 & & & \\
\hline With 43 disks & & 1.1 & 4.3 & $3 / 10$ \\
\hline With 5.5 disks & & 2.2 & 4.8 & $2 / 10$ \\
\hline Experiment 6: & 2.18 & & & \\
\hline With 44 disks & & 2.9 & 3.9 & $0 / 9$ \\
\hline With 22 disks & & 3.4 & 5.2 & $1 / 9$ \\
\hline With 11 disks & & 3.6 & 5.4 & $1 / 9$ \\
\hline With 5.5 disks & & 3.9 & 5.9 & $1 / 9$ \\
\hline Experiment $7: 21$ trees, & 1.90 & & & \\
\hline Optical flow alone & & 0.6 & 2.1 & $11 / 12$ \\
\hline $\begin{array}{l}\text { Optical flow with small oscilla- } \\
\text { tions }{ }^{d}\end{array}$ & & 1.1 & 4.2 & $5 / 12$ \\
\hline Experiment 8: 2 I trees, & 1.90 & & & \\
\hline Optical flow alone & & 0.6 & 1.4 & $3 / 3$ \\
\hline $\begin{array}{l}\text { Optical flow with large oscilla- } \\
\text { tions }{ }^{d}\end{array}$ & & 2.4 & 4.4 & $0 / 3$ \\
\hline Experiment $9: 21$ trees, & 1.90 & & & \\
\hline In a rigid environment & & 0.6 & 2.1 & $11 / 12$ \\
\hline In a nonrigid environment & & 0.9 & 2.8 & $9 / 12$ \\
\hline
\end{tabular}

${ }^{a}$ Velocities are given for an individual with an eye height of $1.6 \mathrm{~m}$.

${ }^{\mathrm{b}}$ Seventy-five percent criterion was suggested by W. H. Warren et al. (1988). We suggest a $95 \%$ criterion instead, roughly allowing collision with every 20 th tree rather than every 4 th. Except in Experiment 6 , criteria were determined for each individual by fitting their data (range transformed in the ordinate and $\log$ transformed in the abscissa) to a logistics curve.

${ }^{c}$ The accuracy needed for a forward velocity of $1.85 \mathrm{~m} / \mathrm{s}$ is $3.97^{\circ}$; for $1.9 \mathrm{~m} / \mathrm{s}, 3.86^{\circ}$; and for $2.18 \mathrm{~m} / \mathrm{s}$, $3.39^{\circ}$. All of these values were computed in a fashion analogous to those in Table 1 .

The oscillations release these conditions from the fixed-camera-angle technique, but because no object is "fixated" (held steady in the middle of the field of view), the conditions are not like the differential motion parallax displays.

is the essential identity of performance on both types of trials. Either decomposition proceeds seamlessly, or it occurs not at all.

Matching errors to DMP failure. A performance level of $95 \%$ at gaze-movement angles of less than $2^{\circ}$ is completely adequate for the task, but consider a not-so-rhetorical question: Why should observers make any errors at all? Even on trials with a final gaze-movement angle of only $0.25^{\circ}$, there is relative motion in the display well above any motion threshold. Moreover, no one has given a systematic account of errors in a wayfinding task.

Cutting (1986) outlined conditions under which DMP should fail and indicated where errors might occur. In particular, movement through some environments may have greater velocities for farther trees than nearer ones, due to stochastic factors of tree distribution, thus violating Equation 10. Figure 8 shows some situations where DMP would fail. Objects 1 are nearest to the moving observer, Objects 2 are at 
Violations of Differential Motion Parallax
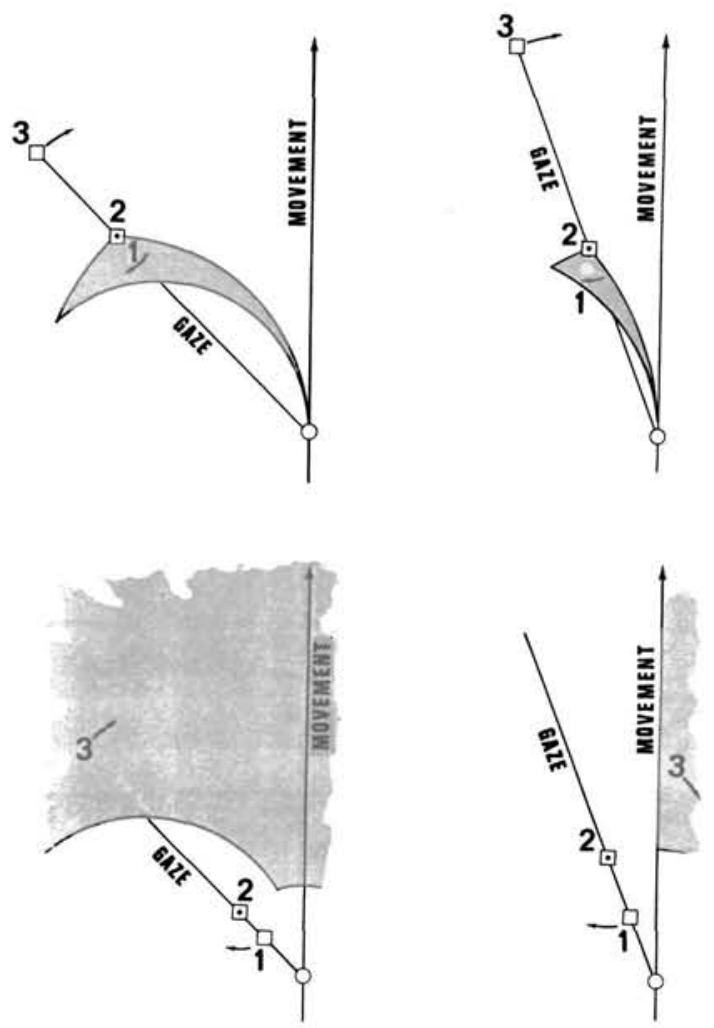

Figure 8. Situations yielding violations of differential motion parallax. (Object 2 is the fixated object, Object 1 is closer than fixation, and Object 3 is more distant. In the top panels Object 3 serves as a reference object and the stippled areas represent all possible locations of Objects 1 violating the inequality of Equation 10 . That is, within these regions a nearer object would move slower than, and in the opposite direction from, a farther object. In the lower panels Object 1 is a reference object and the stippled areas are those locations for Objects 3 that would violate Equation 10 . That is, within these regions a farther object would move faster than [and in the opposite direction from] a nearer object.)

fixation, and Objects 3 are farther away. The top panels show positions where any Object 1 would move slower than, and in the opposite direction from, Object 3; the bottom panels show positions where any Object 3 would move faster than, and in the opposite direction from, Object 1.

Notice further, comparing top-left and top-right panels, that as the gaze-movement angle decreases, there is less area in the foreground in which to place an object to sustain DMP. Indeed, the task of avoidance necessarily removes those objects from near one's path, leaving only the texture on the ground plane, far below the line of sight, to satisfy DMP. Thus, by this account, the act of avoiding one obstacle along one's path actually increases the possibility of a wayfinding error in avoiding a subsequent obstacle.

Most important, however, is the empirical test: If observers were incorrect on trials with DMP violations and correct on those without, this would be strong evidence that viewers actually use DMP for wayfinding. As Cutting (1986) offered no data, what follows is the first test of this possibility.

For all stimuli, coordinates in the $x z$ plane of the six nonfoveal trees were entered into a computer program that calculated their direction and relative velocities during the sequence. All stimuli were then assessed for any violations of DMP across their 54 successive frame pairs. Because trials with and without bounce and sway were matched in tree locations, and because the oscillatory motions do not cause violations of DMP, this investigation was reduced to 28 unique trials, 14 with simulated fixation to the right of observer movement and 14 to the left.

Of these 28 stimuli, 12 were found with DMP violations on at least one pair of frames: One each at $16^{\circ}, 8^{\circ}$, and $4^{\circ}$; two each at $2^{\circ}, 1^{\circ}$, and $0.5^{\circ}$; and three at $0.25^{\circ}$ final gazemovement angle. Six of these 12 stimuli had DMP violations throughout at least the first 44 pairs of frames (two stimuli each at $1^{\circ}, 0.5^{\circ}$, and $0.25^{\circ}$ ). The four stimuli at less than $1^{\circ}$ violated DMP throughout the entire 55-frame sequence, and performance on these four stimuli averaged only $14 \%$ correct. The two stimuli at $1^{\circ}$ had their last 10 pairs of frames revert, following Equation 10, and performance on these stimuli was $70 \%$.

With these considerations in mind, we parsed the data into two groups, those consistent with and those inconsistent with DMP during the final stimulus frames. These groups are shown in Figure 7. Three things are to be noted. First, performance was nearly $100 \%$ throughout for those 16 stimuli without any DMP violations. Second, performance was considerably worse for stimuli with DMP violations whose final gaze-movement angle was $1^{\circ}$ or less. Third, performance on those stimuli with final angles greater than $1^{\circ}$ differed little from performance on those stimuli consistent with DMP. Other possible sources of information are considered in Experiment 2 , but these data strongly suggest that viewers followed the rules of DMP, sometimes to their detriment, within $2^{\circ}$.

To reinforce this result, consider a second analysis. We divided the sequences for all 28 stimuli into quintiles of 10 sequential pairs of frames each and coded them for the presence of Equation 10 violations. We then performed multiple regression using the quintile codes as predictors of performance. Codes for the first 4 quintiles accounted for less than $1 \%$ of the variance (all $p s>.60$ ); codes for the last quintile, however, accounted for $68 \%$ of the variance in observer performance, $F(1,22)=30.9, p<.0001$. Clearly, if there were violations of DMP at the end of a stimulus sequence, it was these violations and no others that affected performance.

Finally, to demonstrate the potency of DMP in these data, the final gaze-movement angles and the fifth quintile DMP codes were regressed against observer performance. The partial correlation of gaze movement angle against performance was not reliable, $r=.10, p>.38$, whereas the partial correlation for the fifth quintile code against performance was reliable, $r=.77, p<.0001$. Thus, DMP is a better predictor of performance than even the most potent orthogonal variable in the experimental design. 


\section{Experiment 2: DMP, Gait, and Route Selection in Continuous and Discontinuous Environments}

We had six goals in Experiment 2. First, we wished to replicate the previous study on a more powerful computer, again looking at any possible differences between stimuli with and without bounce and sway. Second, we hoped to change the task by simulating observer fixation farther into the distance, outside the distance range of Table 1. This changes the setting from one of avoidance of near objects to one of route selection within a particular environment. That is, by looking out into the distance an observer looks for objects to be avoided significantly in the future to pick the best path. Third and fourth, we wanted to observe the effects, if any, of more realistic stimuli and of variation in the number of objects in the environment. Fifth, because DMP is easily described in both discontinuous environments (those without uniformly smooth variation in surfaces, hence with occlusions) and in continuous environments, we generated stimuli reflecting both. Thus, some simulated environments had trees and some had flat disks lying on the ground plane. Finally, we allowed participants to terminate a trial when they felt they had sufficient information, allowing analyses of reaction time and stimulus information immediately before their response.

\section{Method}

Stimuli. Stimuli were generated on-line using a Personal Iris Workstation (Model 4D/20G), with a noninterlaced raster-scan res- olution of $1,280 \times 1,024$ pixels. Four kinds of stimuli were generated for four different conditions, two with small trees and two with colored disks lying flat on a ground plane, as suggested in Figure 9. Each tree was identical in structure. A "forest" was created by translating this tree, replicating it many times across the $x z$ plane, and rotating it randomly around its $y$-axis in each new location. The major branching of limbs occurred at 1.5 eye heights, and each tree was 2.7 eye heights tall. The disks were 1.87 eye heights in diameter, large enough to diminish spatial and motion aliasing near the computed horizon without covering too much of the ground plane. The stochastic arrangement of disks matched that for the trees.

A fixation object, one tree or disk, appeared at the center of the screen at a distance of 31.3 eye heights $(50 \mathrm{~m})$, with the visible horizon clipped at 62.5 eye heights $(100 \mathrm{~m})$. This fixation object was colored a light purple. The ground plane was brown, the sky was blue, and the other trees or disks were light gray. No trees had leaves, so the stimulus was like a sparse winter scene. Near trees occluded far trees. creating discontinuous displays in the motion sequence; although disks often overlapped, they represented a continuous surface.

In two conditions, in addition to the fixation object there were four other objects, trees in one condition and disks in the other; in the other two conditions there were many more trees or disks, with a mean of $74,(S D=2.4)$. These were called the few-tree, few-disk, many-tree, and many-disk conditions. Layouts for the few- and manyobject conditions are shown schematically in Figure 10. New coordinate positions were generated for each trial for each participant. For those conditions involving few trees and disks, the $x z$ coordinates of the nonfixated trees were stored for further analyses. (For the many-tree and many-disk conditions, there were simply too many objects to keep track of, so none were stored.)

Motion was generated by successive frames, locked to the rates of

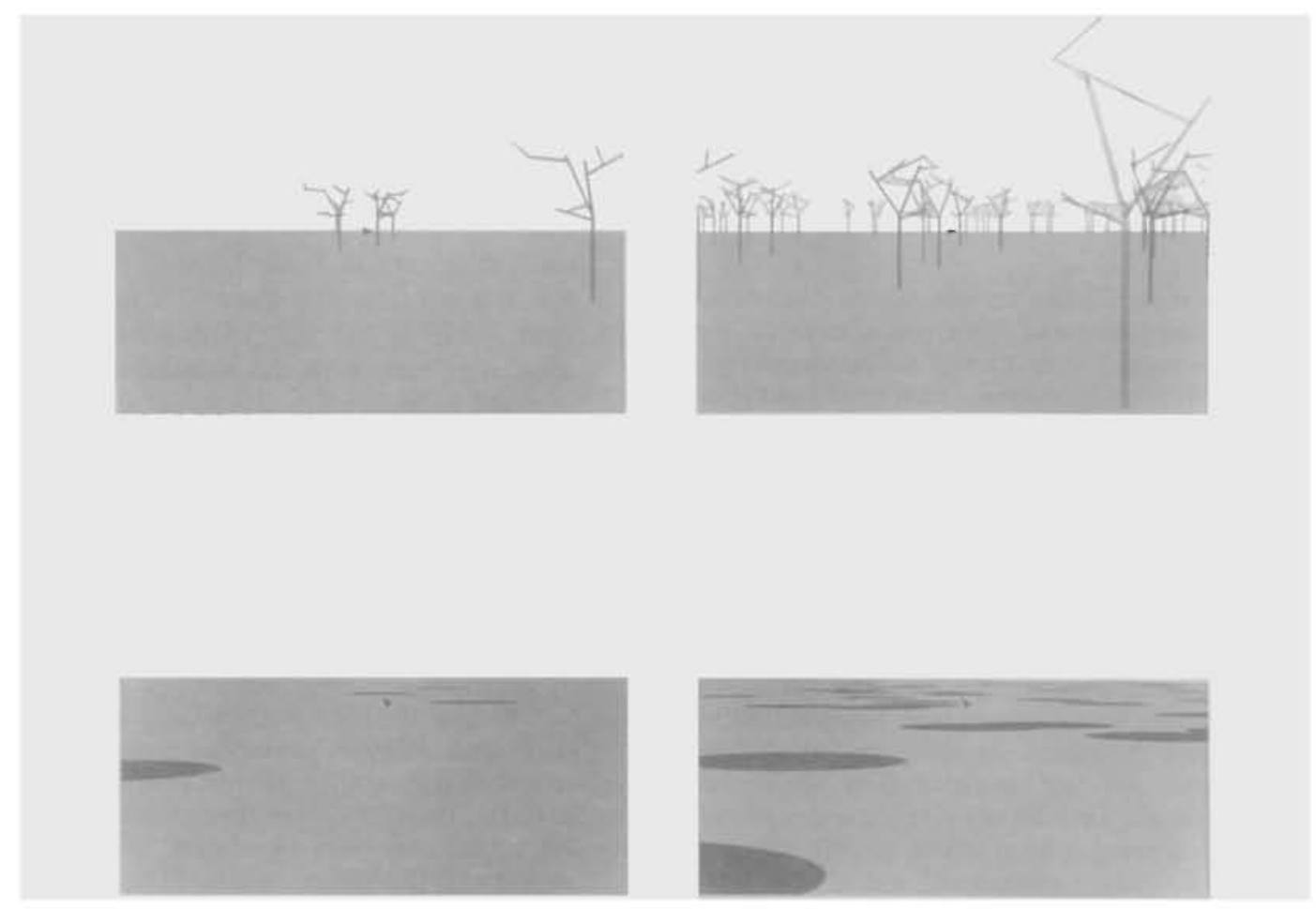

Figure 9. Sample frames from the four conditions in Experiment 2, with few and many trees and disks. (The fixation object is indicated by a small arrow: in the experiment no arrow was present. The ground plane was brown, the trees and disks were gray, and the sky was blue.) 


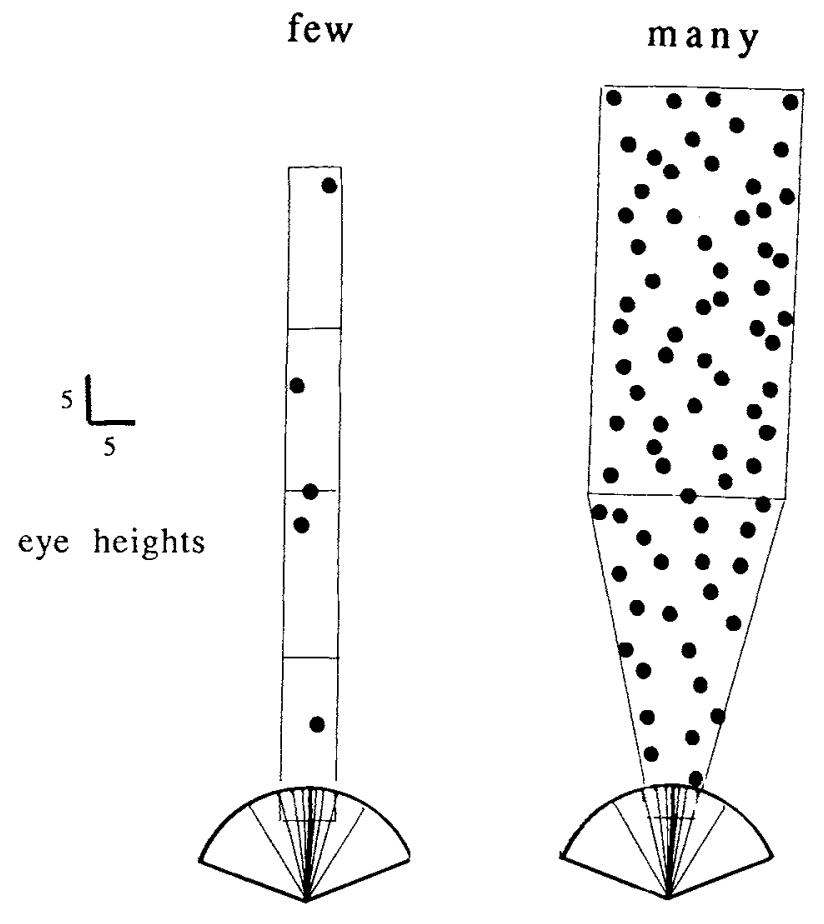

Figure 10. A bird's-eye view of the environments and paths in Experiment 2. (Placement of trees and disks in the few-object conditions is suggested in the left panel; one object was always placed at fixation in the center of the screen, and the four others were placed randomly in each of the four areas, two in front and two behind. Placement of trees and disks in the many-object conditions is suggested in the right panel; again 1 was always placed at fixation in the center of the screen, and the others were placed randomly, 1 on each of 100 slices orthogonal to the line of gaze, 50 behind the fixation object, and an average of 24 visible in front of it. The fans at the bottom suggest the paths taken should the trial progress to its end. Participants were able to terminate a trial; thus, the entire length of each path was rarely taken.)

the displays whose rendering (the amount of time taken to color all pixels) was most time consuming (those with a mean of 74 disks). Thus, all stimuli averaged $220 \mathrm{~ms} /$ frame,${ }^{17}$ the best frame rate available at the time. Without intervention, trials would end after $21 \mathrm{~s}$, but participants were allowed to terminate the trial whenever they felt sure of their response. Thus, reaction times were recorded.

Simulated forward velocity was 1.36 eye heights $/ \mathrm{s}(2.18 \mathrm{~m} / \mathrm{s})$, a bit faster than in Experiment 1 . As the trial progressed trees could disappear off the edge of the screen, due to either simulated forward movement or the shearing motions of DMP. Again, viewing was unconstrained and binocular, but participants were encouraged to look at the fixation object and sit $0.5 \mathrm{~m}$ from the screen, creating a resolution of 50 addressable units (pixels) per degree of visual angle. Perspective calculations for the stimuli were based on this viewing distance. Again, half the stimuli simulated smooth dollying and panning movements, and half simulated these movements plus bounce and sway. Bounce was $6.5 \%$ of eye height and sway was $5.0 \%$; both values are in the upper range of Muray's (1967) measurements for natural gait, providing a more severe test of both DMP and decomposition. A step cycle took $830 \mathrm{~ms}$ to complete. Initial gaze movement angles were $0.125^{\circ}, 0.25^{\circ}, 0.5^{\circ}, 1^{\circ}, 2^{\circ}, 4^{\circ}, 8^{\circ}, 16^{\circ}, 32^{\circ}$, and $64^{\circ}$; median final gaze-movement angles were $0.13^{\circ}, 0.27^{\circ}, 0.55^{\circ}$, 1. $1^{\circ}, 2.2^{\circ}, 4.3^{\circ}, 8.4^{\circ}, 16.6^{\circ}, 32.6^{\circ}$, and $64.4^{\circ}$. These final values are close to the initial ones because of the great distance of the fixation object and the self-termination of trials. For simplicity's sake we use the former as the independent measure.

Presentation order was random for the 80 stimuli per condition: 10 final gaze movement angles $\times 2$ gaze directions (left and right of movement) $\times 2$ carriage conditions (with and without bounce and sway) $\times 2$ replications (with different coordinates for nonfoveated objects). Unlike Experiment 1, each stimulus was generated on-line for each condition for each observer.

Procedure. Eight different members of the Cornell community participated in three sessions, each lasting about $1.25 \mathrm{hr}$. Within a session, they participated in four conditions, with few and many trees and disks. Order of participation was counterbalanced across participants. Each was paid $\$ 20$ for participation. Instructions were the same as those in Experiment 1, but participants pressed the left button of the Iris mouse if they felt they were gazing left of the path of movement and the right button if they felt they were gazing to the right. Again, no feedback was given and no eye movements were monitored. Each participant was given several practice trials before a session began. Viewers again sat in a moderately lit room and the edges of the screen were clearly visible.

\section{Results and Discussion}

The overall patterns of results are shown in Figure 11. Each panel shows the main effect of final gaze-movement angle, $F(9,63)=124.2, M S_{\mathrm{e}}=114.8, p<.0001$. The left panel shows that performance was better for displays with trees than for displays with disks $F(1,7)=9.2, M S_{e}=8.7, p<.02$. The middle panel shows better performance for many objects than for few, $F(1,7)=14.7, M S_{e}=5.3, p<.006$. The right panel shows essentially equal performance for trials with and without bounce and sway, $F(1,7)=2.6, M S_{\mathrm{e}}=2.2, p>.15$. There was also a reliable effect of session, $F(2,14)=3.9, M S_{e}$ $=1.57, p<.044$, with mean performances of $78.2 \%, 79.6 \%$, and $80.6 \%$ across the three sittings.

The median $75 \%$ and $95 \%$ thresholds were $1.3^{\circ}$ and $6.3^{\circ}$, respectively, across the four conditions, but for the manytrees stimuli they were $1.1^{\circ}$ and $3.2^{\circ}$. Values for other conditions are shown in Table 3 . Given that this is a route-finding test, it is not clear how these $95 \%$ values should be compared with Table 1 , but $3.2^{\circ}$ is about the value needed for an avoidance task with the simulated observer velocity used (about $2 \mathrm{~m} / \mathrm{s}$ ).

Because the positions of the four nonfoveal objects were recorded in the few-tree and few-disk conditions, calculations could be made concerning presence and absence of DMP. Moreover, because participants could terminate the trials at any point the information immediately before their voluntary response could be assessed. The left panel of Figure 12 shows

\footnotetext{
${ }^{17}$ Because the Iris is a Unix-based system, stimulus frame rates (how often different information is computed) can vary, although refresh rates (how often different information is displayed) are fixed at $60 \mathrm{~Hz}$. Thus, for $220 \mathrm{~ms} /$ frame, occasionally one frame of motion will appear only every 14 refreshes of the screen, but the mean is closer to 13. In addition, one should be concerned about the motion seen with such slow frame rates. Our retort, however, is twofold: First, the visual motion is very slow at pedestrian speeds, and second, Experiments 8 and 9 show similar results with frame rates nearly four times as fast.
} 

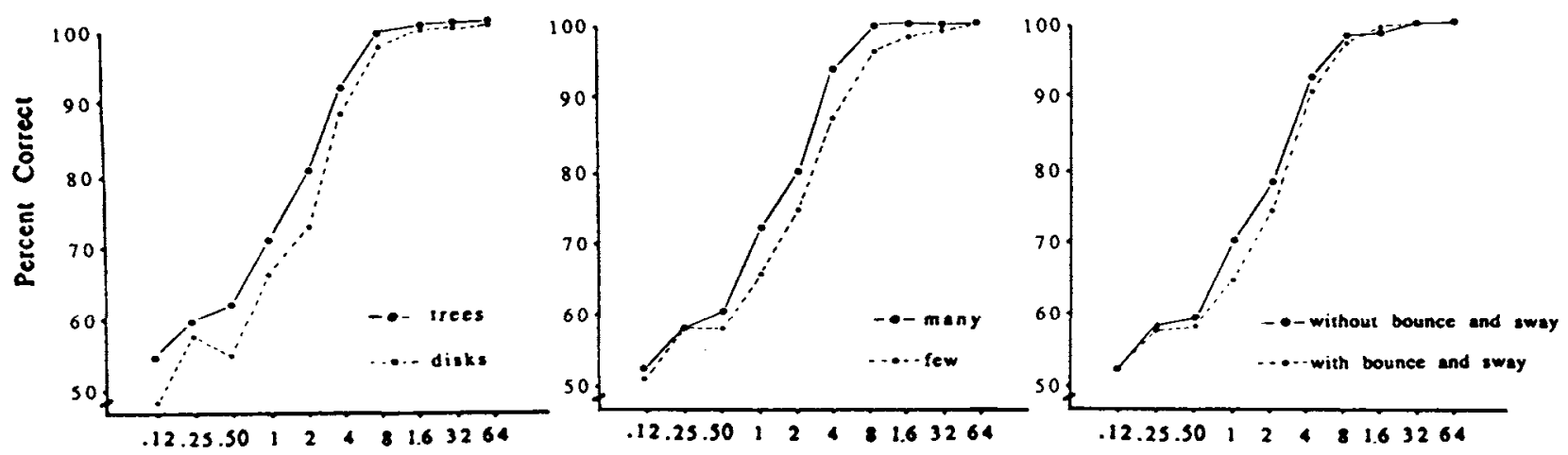

Final Gaze-Movement Angle

Figure 11. Performances across the various combinations of stimuli and conditions in Experiment 2, as a function of final gaze movement angle.

the performance on trials consistent and inconsistent with DMP (an inconsistent trial had motion of trees or disks that violated Equation 10 in its frames immediately before the response). The left panel of Figure 11 also shows residual information being used by observers for gaze-movement angles between about $2^{\circ}$ and $16^{\circ}$, a pattern similar to that seen in Figure 7. That is, even for the trials that are inconsistent with DMP, performance is increasingly accurate. After long scrutiny of the relative retinal motion of near trees and disks, we discovered a typical pattern orthogonal to DMP.

When an observer looks out into the environment at an object deviating $2^{\circ}$ to $16^{\circ}$ from his or her path of movement, several things can happen in the retinal array. The panels of Figure 13 show how the territory can be divided around the fixated object. ${ }^{18}$ Consider the central panel, with a gaze angle of $16^{\circ}$ to the right of the path of movement, and proceed counterclockwise starting from the position of the observer.

First, some near objects to the right side of gaze move outward in the retinal array from the fixation, but in a decelerating manner. This pattern is due to decreasing dominance of the optical (exteroceptive, or forward movement) vector field over the rotational (proprioceptive, or eye movement) field. This outward deceleration (OD) phase is indicated by the stippled region in Figure 12. Second, generally farther objects have come to a halt and have begun to move toward the plane of sight (the vertical plane through the eye and fixated object). This pattern shows the vectors of the rotational flow dominating optical flow. This inward motion (IM) phase is indicated by the white region. Third, the distant, inwardly moving objects cross the plane of sight, in which case objects revert to OD. Finally, some near objects to the left of fixation move inwardly as well.

Notice two things, First, as shown in all the panels of Figure 13, OD and IM can occur for both near and far objects. Thus, for these sources of information to be useful, depth must be computed for each of the objects under question. This requirement might appear to contrast with DMP, for which no depth information is needed because it falls out as part of the calculation (see Equation 10). Second, positions of near ob- jects satisfying DMP can occupy regions of either OD or IM. Thus, DMP is information potentially orthogonal to these two other sources. Indeed, across the 3,840 trials in the fewobject data sets, there was essentially no correlation between the presence of DMP and either of these other sources of information, $r--.010$ for OD and $r=.015$ for IM). Interestingly, these two extra sources were also uncorrelated with each other, $r=-.08$. Thus, it is fairly straightforward to observe the relative and additive effects of these three sources of information.

The issue at stake, then, is the residual information provided by $\mathrm{OD}$ and IM beyond that provided by DMP. ${ }^{19}$ As mentioned earlier, the left panel of Figure 12 shows the effects of DMP as a function of gaze movement angle. When inward acceleration is added to DMP, and one observes performance on trials consistent with either DMP or inward acceleration, most of the residual information is removed, as shown in the middle panel of Figure 12 . That is, only at $8^{\circ}$ is performance above chance for trials in which both of these sources of information fail. When OD is added to DMP and IM the pattern improves even further, as shown in the right panel of Figure 12. That is, at no gaze-movement angle was performance better than $40 \%$ on trials that were inconsistent with these three sources of information. Indeed, performance was always reliably below chance. Remember, however, that these results are for the few-object conditions only. In the manyobject conditions, DMP could dominate simply because of the greater number of objects populating the environments.

\footnotetext{
${ }^{18}$ See also Rieger (1983) and Warren, Mestre, Blackwell, and Morris (1991) for descriptions of motion during circular translation.

${ }^{19}$ We also considered a possible artifact in the stimuli. It could have been that participants simply observed the movement of the object farthest in the periphery of the stimuli and made judgments on the basis of that pseudoinformation. That is, if the most peripheral object on the screen moved left, they responded left; if it moved right, they responded right. This variable accounted for less than $1 \%$ of the variance in the data in Experiments 1 and 2.
} 

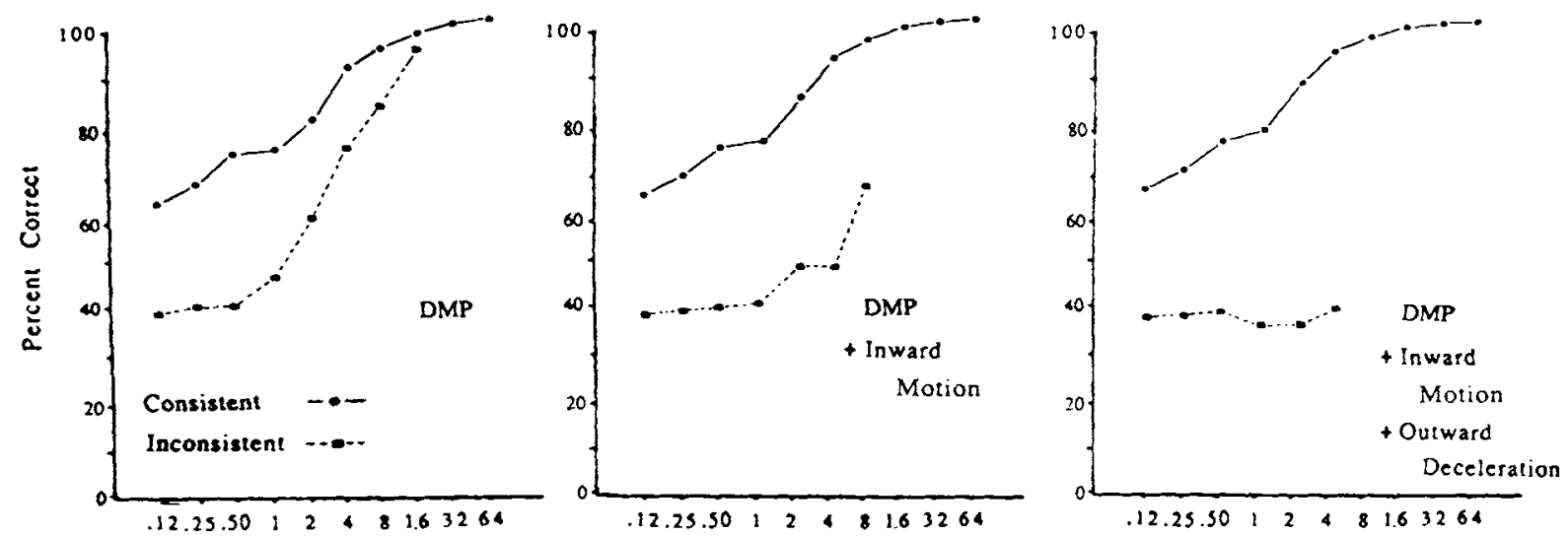

Final Gaze-Movement Angle

Figure 12. The three panels compare performance in Experiment 2 on trials consistent and inconsistent with differential motion parallax (DMP) and the sequential addition of two other retinal sources of information, inward motion and outward deceleration. (Inconsistency is defined as in Figure 8. Inconsistent functions are truncated at points when their continuation would represent less than $5 \%$ of all trials at that gaze-movement angle.)

In natural settings, especially those with rich ground texture, DMP may be all that is needed.

Consider one more aspect of Figure 13. These simulation data show very little area nearby where inward motion is manifested for gaze-movement angles of less than $2^{\circ}$, but quite a lot of area where inward motion can occur beyond fixation; at $16^{\circ}$ there is slightly more area where nearby inward motion can occur; and at $64^{\circ}$ inward motion in nearby regions is substantial. Given that inward motion occurred on a trial, the probabilities that it occurred closer than fixation are $1 \%$, $9 \%$, and $50 \%$ for $2^{\circ}, 16^{\circ}$, and $64^{\circ}$ gaze-movement angles, respectively. Any increment in wayfinding performance is most likely due to the registration of inward motion from objects more distant than the fixation object. Thus, DMP is information about near objects and IM is information largely about far objects. Perrett, Harries, Mistlin, and Chitty (1990) have presented evidence for the existence of cortical neurons responsive to inward motion.

\section{A Second Look At DMP Failures in Experiment 1}

Given the analysis of the relations of DMP, OD, and IM for stimuli in Experiment 2, multiple regression was performed on the stimuli and data of Experiment 1, using these three sources of independent variables. Results were similar. As found previously, DMP was a strong predictor of performance, $F(1,23)=118.4, p<.0001 ; \mathrm{IM}$ also predicted performance, $F(1,23)=74.2, p<.0001$, accounting for the near perfect scores on four stimuli in Figure 7 at final gazemovement angles of $2^{\circ}, 4^{\circ}$, and $16^{\circ}$. However, OD did not

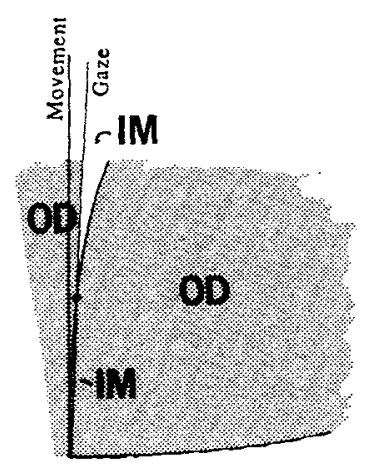

$2^{\circ}$

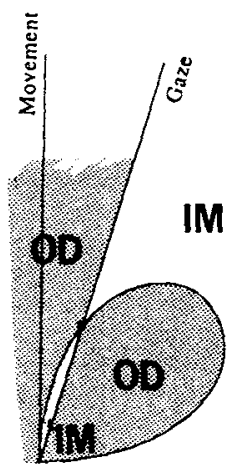

$16^{\circ}$

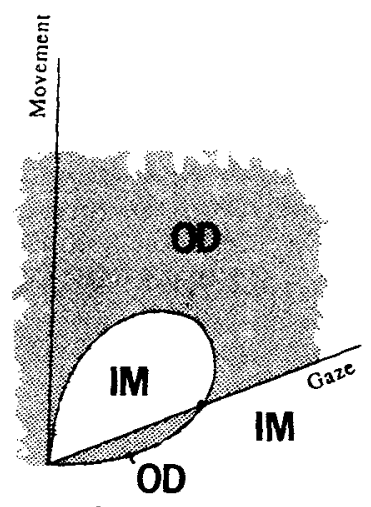

$64^{\circ}$

Figure 13. Given a fixated object $2^{\circ}, 16^{\circ}$, or $64^{\circ}$ to the right of a linear path of movement, the stippled areas show where objects with outward deceleration (OD) would lie, and the unstippled areas show where objects with inward motion (IM) would lie. (Fixated objects are marked with a dot.) 
account for any systematic variance, $F(1,23)=0.97, p>.33$. Performance on all trials ending with inconsistent information about DMP and IM was only $23 \%$. These results fit quite snugly with those of Experiment 2.

\section{Reaction Time and Ordinal Information in DMP}

Despite its strength here in accounting for correct and incorrect responses, wayfinding by DMP as presented previously (Cutting, 1986) had at least two problems. First, Warren and Hannon $(1988,1990)$ properly characterized DMP as a saccadic and a pursuit eye movement theory. They then showed experimentally that saccadic eye movements were not necessary for wayfinding, at least within about $4^{\circ}$. Second, Warren and Hannon properly noted that DMP in our nominal-direction task could provide only nominal information (left or right) about the direction of movement with respect to gaze. They then used a simulated pursuit fixation technique and showed experimentally that observers could be accurate to within about $3^{\circ}$ of visual angle, demonstrating at least some ordinal, or better, information.

We undertook Experiment 2, in part, to analyze stimulus motions and observer reaction times in an attempt to coun- termand these two important problems. Perhaps there is ordinal information within a single pursuit fixation that is adequate for the task.

In addition to the DMP inequality expressed in Equation 10 , we felt the retinal array could contain other information for wayfinding. One contender we favor is the absolute velocity of the fastest moving object. The inset in Figure 14 shows a method for sampling locations in searching for the velocities for such objects. For a series of locations nearer than the fixation object (at means of $90 \%, 30 \%$, and $10 \%$ of the distance to the object), and at various gaze movement angles, a family of curves is generated, as shown in the left panel of Figure 14. When the log-scaled values of these velocities are compared against the median reaction times for responses, shown in the right panel for these same gaze movement angles, three striking correlations are obtained, all $r \mathrm{~s}>97$; all $t \mathrm{~s}(8)$ $>11.3$, all $p<.0001$.

As should be clear from the reaction time data, there was a reliable effect of final gaze-movement angle, $F(9,63)=34.2$, $M S_{e}=718.7, p<.001$, and coupled with the performance data the reaction time data are in the direction opposite from a speed-accuracy trade-off. There was also a reliable decrease in reaction times across sessions, $F(2,14)=8.2, M S_{\mathrm{e}}=680.0$, $p<.005$, with means of $6.3 \mathrm{~s}, 5.0 \mathrm{~s}$, and $4.3 \mathrm{~s}$. There was no
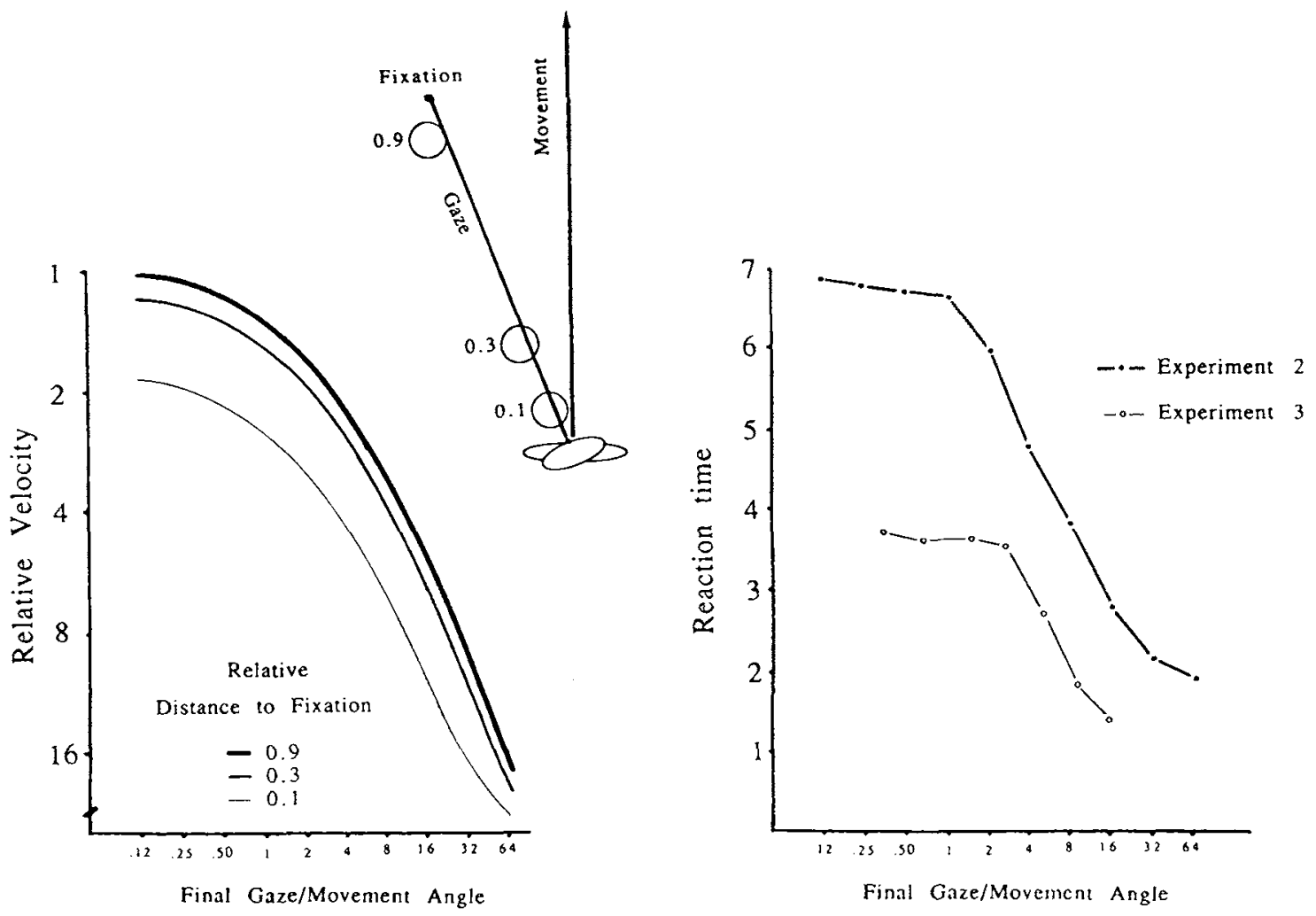

Figure 14. Reaction time analyses promoting ordinal rather than nominal (left vs. right), information in differential motion parallax. (The inset shows a consideration of locations of objects in an environment near a fixated object, $10 \%, 30 \%$, and $90 \%$ of the distance to the object at mean deviations of $3^{\circ}$ from the line of gaze. The left panel shows the velocities [log scaled] at these three points, and the right panel shows the median reaction times [in seconds] in Experiments 2 and 3. Correlations of reaction time with mean retinal velocities are very high, all $r s>.97$.) 
reliable difference between stimuli with and without bounce and sway, $p>.40$, with means of $5.19 \mathrm{~s}$ and $5.24 \mathrm{~s}$, respectively. Slightly longer reaction times might have been expected for bounce and sway stimuli, because the step cycle took 830 ms to complete and added four additional flow fields to the display (see Equation 11).

In summary, the speed of a viewer's response is strongly related to the absolute DMP velocities in the display, suggesting that velocity information is both used by and important to the viewer. Thus, counter to W. H. Warren et al.'s (1988) conclusions about DMP, there may be more than nominal (left vs. right) information in retinal flow that is satisfactory for determining the instantaneous angular distance of gaze from the path of movement; there appears to be ordinal information (relative velocity) as well. The addition of bracketed material in Table 2 updates DMP. The table can now represent a non-saccadic-eye-movement theory, and the rules need not be recursive to make an accurate aimpoint estimate.

Future research will focus on comparisons of the nominaldirection task with other tasks, such as the probe technique used by Warren et al. (1988) and used here in Experiments 4 through 9 for optical flow displays. Future research will also explore any possible causal relation between reaction times and absolute velocity in the retinal field.

\section{Experiment 3: Avoidance Reaction Times to DMP During Gait}

The reaction times shown in Figure 14-up to $7 \mathrm{~s}$ - are quite out of the ordinary for experimental psychology. They are considerably longer even than those found by the Road Research Laboratory (1963) and by Probst et al. (1984) for avoiding traffic accidents; moreover, they are problematic for a particular assumption we made in Table 1 . That is, the range of 4-6 s for gaze-movement angles of $2-8^{\circ}$ would make the reaction time distances burgeon still further and force even more exquisite wayfinding abilities than those already outlined in Table 1. To be sure, a directional foot plant and a finger press on a computer mouse are different actions, but given this range of reaction times, the roles of effector, dexterity, and neural conduction time ought to be minimal.

The reaction times of Experiment 2, however, may reflect several factors, only one of which is pertinent to the calculations in Table 1. In particular, when the display first came up on the screen, the viewer may have spent a certain amount of time simply orienting to its unique features, something which never happens in the natural environment for an alert moving observer. (Imagine running through an environment with eyes closed and suddenly opening them). Thus, to counteract the possible need for orientation time, the first frame of each stimulus display in Experiment 3 was presented for 1 $s$ before the motion sequence began. In addition, the reaction times in Experiment 2 were for a route-finding task with distances out of the range of those considered in Table 1. Thus, the distances were changed here to make them comparable to Experiment 1.

\section{Method}

The stimuli, equipment, and methods were like those in the fewtree condition of Experiment 2, but distances and initial gaze movement angles were the same as those in Experiment 1. Bounce was $4.0 \%$ of eye height and sway was $3.5 \%$; both values are smaller than in the first 2 experiments and closer to the midrange of Murray's (1967) data. A step cycle took $940 \mathrm{~ms}$. Through optimization procedures of simplifying tree shape (but not by reducing the number of branches), presentation rate was increased to $80 \mathrm{~ms} /$ frame. Forward velocity was 1.2 eye heights $/ \mathrm{s}$ (or about $1.9 \mathrm{~m} / \mathrm{s}$ ).

Twelve different members of the Cornell community participated in this experiment (and also in Experiments 7 and 9). Viewers were told to do as well as they could and to respond only when they were sure of their choice. Thus, accuracy, but not speed, was emphasized. This seemed appropriate as a wrong choice of direction in the real world would be costly. The battery of experiments took about $1.5 \mathrm{~h}$ to complete, and viewers were paid $\$ 10$ for their participation.

There were 28 randomly ordered stimuli per block: 7 initial gazemovement angles $\left(0.25^{\circ}, 0.50^{\circ}, 1^{\circ}, 2^{\circ}, 4^{\circ}, 8^{\circ}\right.$, and $\left.16^{\circ}\right) \times 2$ directions of gaze with respect to movement (left or right) $\times 2$ carriage conditions (with and without bounce and sway). Median final gaze movement angles were $0.37^{\circ}, 0.66^{\circ}, 1.4^{\circ}, 2.8^{\circ}, 5.2^{\circ}, 9.5^{\circ}$, and $18.3^{\circ}$; these are used as the independent measure. Viewers participated in five consecutive blocks with no break, but only the last four blocks were scored, totaling 112 trials.

\section{Results and Discussion}

Again, there was a main effect of performance according to final gaze-movement angle, $F(6,66)=48.6, p<.0001$, and again there was no difference between stimuli with and without bounce and sway. The overall median $75 \%$ and $95 \%$ criteria performances were $1.4^{\circ}$ and $4.0^{\circ}$, respectively, somewhat worse than Experiments 1 and 2. Values for the separate conditions are given in Table 3.

More important, the technique of presenting a static frame before the motion sequence reduced reaction times considerably. Median reaction times are shown in the right panel of Figure 14, alongside those of Experiment 2. Reaction times also showed a main effect of final gaze-movement angle, $F(6$, $66)=79.7, p<.0001$, and no effect of carriage. Correlations with the three computed velocity functions shown in the central panel are highly significant, all $r \mathrm{~s}>.98$, all $t \mathrm{~s}(5)>$ 10.8 , all $p \mathrm{~s}<.0001$.

Of most interest is the finding that the interpolated reaction time for a final gaze-movement angle was $3.86^{\circ}$, the accuracy required for a forward velocity of $1.9 \mathrm{~m} / \mathrm{s}$. That reaction time is $3.08 \mathrm{~s}$, extremely close to the upper estimate of the Road Research Laboratory (1963). We think this result amply justifies our assumption of a 3 -s reaction time interval for wayfinding, as outlined in the introduction.

The somewhat poorer accuracy thresholds here and in Experiment 2 as compared with Experiment 1 are due to the demand character of reaction time experiments. Although accuracy, rather than speed, was stressed in the instructions, the benefits of terminating a trial early and getting through with the experiment were probably too much for participants to ignore. We found considerably better performance in Experiments 8 and 9 for similar stimuli in a nonreaction time task. It may be, however, that in real-life situations this same 
speed-accuracy trade-off occurs. Laboratory experiments simulating an impending collision with objects without the consequences of collision may yield overly optimistic estimates of observer performance.

\section{General Discussion}

On the basis of the first 3 experiments, six conclusions can be drawn. First, DMP, a source of information in retinal flow, is adequate for wayfinding in cluttered environments. At forward velocities of about $2 \mathrm{~m} / \mathrm{s}$, performance was at $95 \%$ for a gaze-movement angle of $2^{\circ}$ in a nonreaction time task (Experiment 1) and of $4^{\circ}$ in two reaction time tasks. The decline in performance in the reaction time tasks seems to be due to a speed-accuracy trade-off induced by experimental demands.

Second, as predicted, the retinal motions caused by the bounce and sway of natural gait had no effect on wayfinding ability. Given the number of additional components added to retinal flow (Equation 10), either decomposition proceeds seamlessly or this result is embarrassing for any decompositional scheme. The current data cannot differentiate the two possibilities; the results of later experiments address this issue.

Third, a theory of wayfinding based on DMP can account for both correct responses and errors. In particular, when there are violations of DMP at the time of response, a moving observer is apt to be incorrect in locating the direction of his or her aimpoint. We take this as strong evidence that DMP is actually used in the experimental situation, and perhaps is used in real life.

Fourth, DMP seems to be the most important of several sources of information in the retinal motion. Of the other sources of information considered, IM accounts for less variance in the data than DMP, and OD accounts for considerably less again. We interpret the fact that multiple sources of information exist and are used as support for directed perception (Cutting, 1986, 1991a, 1991b), in contrast to direct perception (J. J. Gibson, 1979). Directed perception insists on many-to-one mapping between information and any property of an object or event; direct perception, on the other hand, insists on one-to-one mapping (e.g., Burton \& Turvey, 1990). Thus, we believe Gibson not only overestimated the utility of optical flow but also underestimated the potential plethora of motion information.

Fifth, the assumption of a 3-s reaction time interval seems appropriate for a wayfinding task and is generally in line with results from tests of automobile driving. Relative motion information appears to accrue over a long period of time.

Sixth, in response to the important criticisms of W. H. Warren et al. (1988), wayfinding based on DMP need not require saccadic eye movements, only pursuit fixations. Reaction times are well matched to different absolute retinal velocities within a pursuit fixation at different gaze-movement angles. These results indicate the presence of ordinal information (relative velocity) in retinal flow for the location of the aimpoint.

One might think that the conjunction of these reasons is sufficient to negate the efficacy of wayfinding from IOF; but it is not. In particular, directed perception allows more than one source of information for each perceptual property or task. Given directed perception, it could be that DMP, and its associated retinal motions, are among several other possible sources of information for wayfinding, including various aspects of IOF. If so, IOF must be addressed on its own terms. Thus, we needed to perform a second battery of experiments-Experiments 4 through 6-to replicate and extend the methods and results of Warren et al. (1988).

\section{Optical Flow and Its Structure for Wayfinding}

There have been many experiments on accuracies of wayfinding from optical flow. Llewellyn (1971), Johnston et al. (1973), and R. Warren (1976), for example, found wayfinding accuracies of about $5^{\circ}$ or considerably worse. Others have found better performance (Ahumada, 1983; Carel, 1961; Kaufman, 1968; Riemersma, 1981), but in each of these studies edge rate (the relative velocity of objects in the environment against a fixed point, such as a strut or the edge of a cockpit window) could have been used ${ }^{20}$ Larish and Flach (1990) have shown that edge rates can dominate other optical flow variables. Moreover, edge rates are not available to a runner or walker; neither looks at the world through a screen with fixed edges.

Warren and his coworkers (Warren et al, 1988, 1990; Warren \& Hannon, 1988, 1990), however, conquered some of the methodological problems inherent in some of the earlier research and found $75 \%$ and $95 \%$ performance levels at about $1.5^{\circ}$ and $6^{\circ}$, respectively. With the DMP experiments of Cutting (1986), Warren's corpus of IOF research was among the first to come close to meeting the wayfinding requirements listed in Table 1, seemingly without unnatural visual aids. Given the results of the first three DMP experiments, and the conclusions reached from them, it seemed prudent to replicate some of Warren's IOF findings.

\section{IOF Displays Use a Fixed-Camera-Angle Technique}

Research investigating the utility of IOF generally uses a different technique than studies investigating DMP (but see Warren \& Hannon, 1990). We call it the fixed-camera-angle technique. That is, observers are presented with displays simulating the motions in environments that would be seen if a camera were mounted on a smoothly traversing vehicle at a fixed angle to the vehicle's path. Often, that angle is at or near $0^{\circ}$, and the center of the camera image is at or near the aimpoint. In camera terminology, this technique is pure dolly, without pan; the simulated pursuit fixation technique, of course, is a combination of dolly and pan.

\footnotetext{
${ }^{20}$ When edge rates are nulled, a pilot or driver can align the eye with a point on the structure of the vehicle and allow no motion in the environment. This procedure is called the "gunsight" method of flying (Hasbrook, 1975; Langewiesche, 1944). It is known to be very useful to pilots, particularly pilots of light aircraft, where the heading and aimpoint can be very different.
} 


\section{Experiments 4 and 5: Replications of Warren et al. (1988)}

\section{Method}

Stimuli were generated on a Personal Iris Workstation. As in some of the conditions of Experiment 2 (described earlier), these stimuli presented many light gray disks on a brown ground plane, with a blue sky background and a horizon clipped at 62.5 eye heights. Only a linear dollying motion was used, at 1.36 eye heights/s (or 2.18 $\mathrm{m} / \mathrm{s}$ ), with a trial duration of $7.3 \mathrm{~s}$. Stimuli in Experiment 4 had 74 $(S D=2.4)$ disks, as in Experiment 2; stimuli in Experiment 5 had either $43(S D)=2.0)$ or $4.5(S D=1.0)$ disks. W. H. Warren et al. (1988, Experiment 2) used 63,27, 10, and 2 dots on an unseen plane, presented at three velocities, the mean of which was 1.39 eye heights/ $s(M d n=1.19$ eye heights/s), with a horizon clipped at 23.3 eye heights and a trial duration of $3.7 \mathrm{~s}$. Viewers sat such that the screen subtended a $25^{\circ}$ visual angle (measured horizontally) with a resolution of 50 pixels/degree; Warren et al.'s viewers sat closer to a similar screen so that it subtended a $40^{\circ}$ visual angle, but provided only 32 pixels/degree. Optimization of computer programs used in Experiment 2 allowed stimuli to be presented at $100 \mathrm{~ms} /$ frame; they were presented at $67 \mathrm{~ms} /$ frame by Warren et al.

Warren et al.'s (1988) best technique, a postmotion probe, was used in Experiment 4. That is, the trial sequence proceeded to its end, the last frame was keep on the monitor, a probe appeared at the horizon, and the task of the participant was to determine if the probe were to the left or right of the aimpoint. In Experiments 4 and 5, participants responded with the left or right button on the Iris mouse. Trials could not be terminated by the viewer, so no reaction times were recorded.

In Experiment 4, the 4 locations of the probe and the $37(40-3$ duplicates) aimpoint locations were identical to those used by Warren et al. (1988, Experiment 2 ); that is, probes were $2^{\circ}$ or $6^{\circ}$ left or right of the center of the screen, and aimpoints were $0.2^{\circ}, 0.5^{\circ}, 1^{\circ}, 2^{\circ}$, and $4^{\circ}$ left or right of the probe. This yielded 40 stimuli, which were replicated and randomly intermixed for a total of 80 trials.

In Experiment 5, stimuli were generated with few (4.5) and many (43) disks, with 13 probe locations $\left(0^{\circ}, 1.2^{\circ}, 2.4^{\circ}, 3.6^{\circ}, 4.8^{\circ}, 6.0^{\circ}\right.$, and $7.2^{\circ}$ to the left or right of center screen) and many different aimpoint locations (at $0.125^{\circ}, 0.25^{\circ}, 0.5^{\circ}, 1^{\circ}, 2^{\circ}, 4^{\circ}$, and $8^{\circ}$ to the left or right of the probe). Because some of these eccentricities would place the aimpoint offscreen or very near its edge, the $\pm 8^{\circ}$ aimpoints were not included for probes greater than $3.6^{\circ}$, the $\pm 4^{\circ}$ aimpoints were omitted for probes beyond $4.8^{\circ}$, and the $\pm 2^{\circ}$ aimpoints were also omitted for the $7.2^{\circ}$ probes. This trimmed the number of stimulus trials to 154 . These trials were randomly intermixed for each participant.

Nine different members of the Cornell community served in Experiment 4 . The same 9 , plus 1 additional individual, served in Experiment 5. These 10 also participated in Experiment 6. Order of participation was Experiment 5, then Experiment 6, then Experiment 4. The entire battery of experiments took about $1.5 \mathrm{hr}$. Participants were paid $\$ 10$ each.

\section{Results and Discussion}

Accuracies for Experiment 4 are shown in the upper-left panel of Figure 15, compared with those for W. H. Warren et al. (1988, Experiment 2, 63-dot condition). There is essentially no difference between the two sets of results. The new data show a highly reliable effect of angular separation between probe and aimpoint, $F(4,32)=45.4, M S_{\mathrm{e}}=2,346, p$ $<.0001$. The median $75 \%$ threshold was at $1.5^{\circ}$, and the $95 \%$ threshold was at $6.0^{\circ}$, a little worse than required (see Table 1).

Accuracies for Experiment 5 are shown in the next two upper panels, parsed according to the presence of many and few disks. These accuracies are also compared with the data of Warren et al. (1988, Experiment 2), collapsing across their dense (63 and 27) and sparse (10 and 2) dot conditions. Again the new data show a reliable effect of probe-aimpoint angle, $F(6,54)=78.5, M S_{e}=6,144, p<.0001$, and a reliable effect of disk numerosity, $F(1,9)=10.2, M S_{\mathrm{e}}=1,120, p<.013$. There was also an interaction between angle and numerosity, $F(6,54)=2.8, M S_{\mathrm{e}}=153, p<.011$, reflecting a general superiority of performance on the many-disk stimuli of about $8 \%$ at all angles except $0.125^{\circ}$ and $0.25^{\circ}$, where a floor effect occurred. The median $75 \%$ and $95 \%$ thresholds for the manydisk stimuli were at $1.0^{\circ}$ and $4.3^{\circ}$ (the latter were somewhat poorer than required by Table 1 ); and those for the few-disk stimuli were worse, at $2.2^{\circ}$ and $4.8^{\circ}$, respectively.

Further inspection of these data sets, however, revealed another trend, unreported by W. H. Warren et al. (1988) but analyzed by Warren, Mestre, Blackwell, and Morris (1991) and Warren, Blackwell, and Morris (1989), and reported earlier by Llewellyn (1971) and Johnston et al. (1973). That trend is the systematic bias to report the aimpoint nearer to the screen center than it actually is. To introduce this concept, consider the third function in each of the upper panels of Figure 15. These are plots of performance based on a simple rule: Ignore the motion during the trial and simply note the position of the probe on the screen. If the probe is to the right of screen center, report the aimpoint to the left; if the probe is to the left, report the aimpoint to the right.

Reflections of this bias are shown in the bottom left and right panels of Figure 15 for Experiments 4 and 5, respectively. (The data shown for Experiment 5 omit the $0^{\circ}$ probe and to reduce noise are collapsed across probes at $1.2^{\circ}$ and $2.4^{\circ}, 3.6^{\circ}$ and $4.8^{\circ}$, and $6.0^{\circ}$ and $7.2^{\circ}$ eccentricities.) In particular, performance for probes nearest the center of the screen was better than for probes nearer the edge of the screen, $F(1,8)=7.7$, $M S_{\mathrm{e}}=10.0, p<.024$, for Experiment 4; and $F(1,45)=54.9$, $M S_{\mathrm{e}}=53.2, p<.0001$, for the linear trend in Experiment 5 for probe-aimpoint angles of 0.25 through $2^{\circ}$ only.

One way to assess the relative strength of this bias is through multiple regression using two independent variables: probeaimpoint direction (the experimental variable of interest) and position of the probe on the screen (inducing bias left or right). In other words, the latter variable would work if the viewer completely failed to watch during the trial and responded opposite to the position of the probe on the screen. Partial correlations showed that both variables were reliable predictors of the data across subjects; in Experiment 4, $r=$ $.80, p<.001$, for probe-aimpoint direction; and $r=.20, p<$ .05 , for probe position on the screen. In Experiment 5 , for the dense displays probe-aimpoint direction was about the same strength as the bias, $r s=.53$ and .45 , respectively, $p s<$ .01 , and for the sparse displays the strength of the probeaimpoint direction was exceeded by the bias, $r s=.40$ and .63 , $p s<.01$.

The presence of bias is not good news. It means that, in considerable part, viewers use the available frame of the screen 


\section{EXPERIMENT $4 \quad$ EXPERIMENT 5}
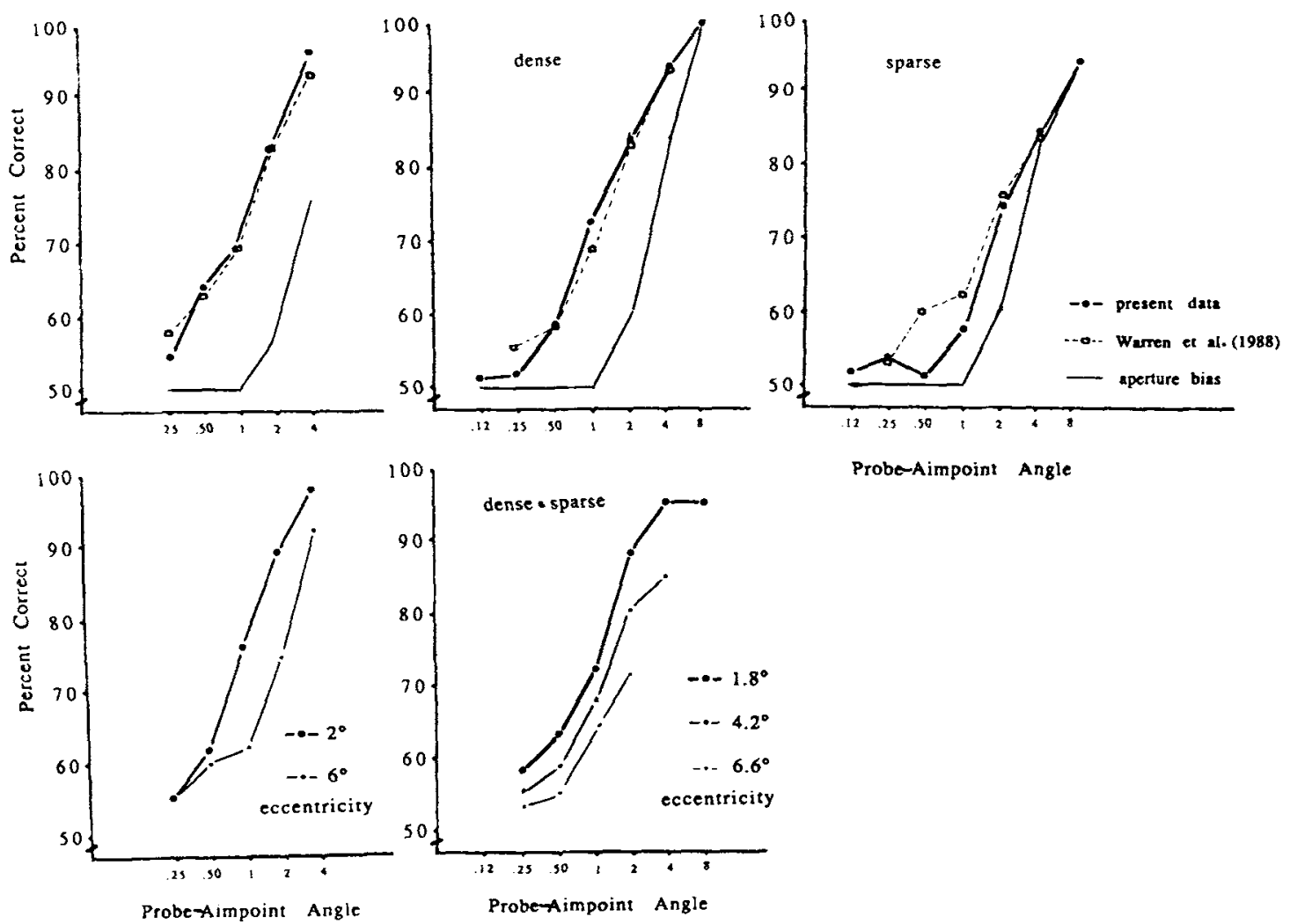

Probe-Aimpoint Angle

Figure 15. Results of Experiments 4 and 5. (The top-left panel compares these results against selected results from W. H. Warren, Morris, \& Kalish [1988, Experiment 2]. The top-left panel compares the data for 74 disks against the 63-dots condition of Warren et al. Superimposed on the data is a performance function based solely on bias, responding only according to the position of the probe on the display. The top-middle panel shows the data from Experiment 5 with a mean of 43 disks against the mean of the 63- and 27-dot conditions of Warren et al.; the top-right panel shows the data of 4.5 disks of Experiment 5 against the mean of the 10- and 2-dot conditions of Warren et al. Superimposed on these data is a performance function for Experiment 5 based again on a bias, responding to probe position alone. The bottom panels show the results of Experiments 4 and 5 parsed according to the eccentricity of the probe location on the display, showing bias. Performance is better when probes are nearest the center of the screen because there is a bias to see the aimpoint at the screen's center.)

in making their judgments. At best, the aimpoint appears to be partly disguised by the aperture (screen) through which a viewer looks. Aperture effects have been found in related areas of visual perception (Perrone, 1980). What is not clear is whether the effect seen here is a perceptual bias (viewers actually "see" the aimpoint displaced toward the center of the screen) or a response bias (in situations of uncertainty they simply respond on the basis of the probe position). Experiment 6 was designed to disentangle these two possibilities.

\section{Experiment 6: A Direct Measure of Aimpoint Estimation From Optical Flow}

Rather than have a probe appear at the end of a trial, viewers were given free reign with the Iris mouse, with the task of moving its cursor to the position of the aimpoint. Our idea was that if the cursor could be superimposed on the aimpoint, this would reduce the chance of response bias in favor of a perceptual bias. Thus, results similar to Experiments 4 and 5 would favor a perceptual bias; different results might favor a response bias in those studies.

\section{Method}

The stimuli were generally the same as those in Experiments 4 and 5. with a block comprising 80 stimuli trials. Nineteen aimpoint positions were used: 0 and $\pm 1.2^{\circ}, 2.4^{\circ}, 3.6^{\circ}, 4.8^{\circ}, 6.0^{\circ}, 7.2^{\circ}, 8.4^{\circ}, 9.6^{\circ}$, and $10.8^{\circ}$, with the central position represented twice as often as the others. Four disk densities were used in the display, with means of $5.6,11.1,22.2$, and 44.5 disks per display; standard deviations were $0.6,1.2,2.5$, and 5.0. The same 10 participants as in Experiment 5 viewed the displays. They were told to move the cursor (a red, slanted arrow) over the aimpoint and to respond by pushing the left mouse 
button. The viewer's response terminated the trial, unless $7.1 \mathrm{~s}$ went by, in which case the screen froze, waiting for the response. Of most interest is the unsigned error between placement of the mouse and the true aimpoint on the screen; unsigned errors ignore whether mouse placement is to the left or the right.

\section{Results and Discussion}

The mean of median unsigned errors across subjects was $1.92^{\circ}$. (The mean signed error was $0.15^{\circ}$.) There was a reliable effect of the number of objects in the display, $F(3,27)=10.7$, $M S_{\mathrm{c}}=29.9, p<.001$, with mean unsigned errors of $2.7^{\circ}$, $2.4^{\circ}, 2.1^{\circ}$, and $1.8^{\circ}$ with increasing numbers of disks. The median $75 \%$ and $95 \%$ criteria thresholds for the 44 disk condition were $2.7^{\circ}$ and $3.9^{\circ}$, respectively, roughly comparable to Experiments 4 and 5 .

There was also a main effect of aimpoint eccentricity, $F(8$, $72)=4.98, M S_{\mathrm{c}}=8.54, p<.001$. Collapsing across left and right aimpoints, the center biases were $0.93^{\circ}, 0.88^{\circ}, 1.03^{\circ}$, $1.28^{\circ}, 1.50^{\circ}, 1.42^{\circ}, 1.62^{\circ}, 2.24^{\circ}$, and $2.27^{\circ}$, respectively for the 9 noncentral aimpoint positions from $1.2^{\circ}$ to $10.8^{\circ}$. These results suggest that the bias is perceptual rather than the result of some kind of response compensation in ambiguous displays. The cause of this aperture effect is not clear, but it may have to do with an interaction of edges (and hence edge rates) with the movement of individual disks. Unfortunately, there is a contamination worse than perceptual bias in Experiments 4 through 6 .

\section{Pixel Creep}

In the course of conducting Experiments 4 through 6 , it became clear to us there was another source of information viewers could use to perform the task. We call this information pixel creep; Rieger and Toet $(1985$, p. 378$)$ and many others before them have called it staircasing. It is the discrete motion of an object due to space-time aliasing in raster-scan displays. (No anti-aliasing measures were taken for stimulus generation here, nor were they taken for the dots stimuli of W. H. Warren et al., 1988.) That is, at 50 pixels/degree of visual angle and $10 \mathrm{frames} / \mathrm{s}$ in these studies (and most likely at 32 pixels/ degree and 15 frames/s in Warren et al., 1988), disks moved in a stepwise fashion across the screen, particularly near the horizon and near the aimpoint.

Rules for aimpoint estimation from pixel creep are straightforward: The faster the lateral stepwise movement and the more it approaches the appearance of continuous motion, the farther the disk is from the aimpoint; also, the slower the stepwise movement, the closer the disk is to the aimpoint. Finally if pixel movement can be counted at fewer than one per second, the disk is quite close to the aimpoint. To accomplish this wayfinding task, then, the observer need only note the rate of pixel creep (the rate at which a dot's or a disk's perimeter discretely moves down and across the screen) for a series of disks or dots relatively near the horizon and interpolate among them to judge aimpoint.

The importance of the pixel-creep artifact in raster scan displays is that it automatically performs retinal flow decomposition and directly presents the viewer with optical flow.
That is, because the environment moves "behind" a discrete grid of pixels anchored to environmental coordinates, the rate of pixel creep becomes a rich source of information about edge rates, which are a dominant force in the perception of optical flow (Larish \& Flach, 1990). Thus, decomposition of retinal flow is accomplished not by the observer but by the display, shortcutting the computational process of reading Equation 4 left to right. ${ }^{21}$

We claim that Experiments 4 through 6, and perhaps the experiments of Warren et al. $(1988,1990)$, are subject to this artifact and that the results gathered in our studies should not necessarily be interpreted to support any natural form of wayfinding. In Experiment 7, we devised a scheme to remove this aliasing artifact through the addition of retinal motions; any decomposition scheme ought to be able to disregard these.

\section{Experiment 7: Minimizing Effects of Pixel Creep by Adding Rotational Flow Fields From Bounce and Sway}

Creating displays that are "antialiased" in both space and time is computation-intensive work. The process involves subpixel addressing and averaging across subpixels to create smooth contours as well as sampling or blurring between successive frames to create smooth motion. Computation of such displays for experimental purposes is beyond the realtime capabilities of the Personal Iris. There is, however, an alternative. By adding certain motions to the display, some aliasing artifacts - such as pixel creep-can be overridden such that they cannot be picked up by the visual system.

Thus, we decided to conquer pixel creep by adding some rotational motions to the optical flow display. The rotational motions we chose, not entirely by coincidence, are those created by counterrotation of the eye during bounce and sway. In other words, updating Equation 11 , we created a visual display of the following:

Retinal flow $\Leftrightarrow$ Rotational flow around the $y$-axis identical to that generated by sway +

rotational flow around the $x$-axis identical to that generated by bounce +

optical flow for linear translation.

Any adequate decomposition scheme ought to be able to remove these complicating rotational flow fields and extract optical flow as the residual. Notice, however, that in such displays eye position itself does not undergo bounce and sway; these optical components have been removed from the equation.

The counterrotational eye movements simulated were very slight, corresponding only to oscillations of $\pm 0.20^{\circ}$ and $0.14^{\circ}$ for bounce and sway, respectively, in Experiment 2; however,

\footnotetext{
${ }^{21}$ Pixel creep is not a methodological problem for DMP displays (those with simulated pursuit fixation) because any differential pixel creep is associated with retinal motion, not optical motion; thus, it cannot aid any decomposition process. Moreover, with bounce and sway motions added, any differences in pixel creep are overridden by much larger (retinal) motions in the display.
} 
they are sufficient to move the horizon and all objects in the display sinusoidally by about \pm 10 pixels vertically and about \pm 7 pixels horizontally. This slow, periodic motion is sufficient to hide left- and rightward pixel creep. It also makes this display one of a non-fixed-camera-angle technique and releases the coordinates of the display from their previous anchor to the environment. The stimuli looked a bit like what would be seen with a rhythmically bouncing camera dollying through the environment on underinflated tires.

Our prediction for the effect of these rotational motions is this: If pixel creep is not a factor confounding wayfinding results using fixed-camera-angle displays, results should be just about the same. Because the jitter is less than one quarter degree, performance ought to be impeded by no more than this amount. If, on the other hand, pixel creep is a factor, performance ought to be considerably worse.

\section{Method}

Stimuli were again generated on the Iris. Each consisted of 21 trees $(S D=2.9)$, with each tree identical to those in Experiment 3. The locations of the trees within the environment were random with no restrictions. The horizon was truncated at 62.5 eye heights, and the field of view was again $25^{\circ}$. Sequences presented each frame for 120 $\mathrm{ms}$, and trial durations were $4.3 \mathrm{~s}$. Subjects could not terminate the trials as they did in Experiments 2, 3, and 6. Simulated forward velocity of the observer was 1.2 eye heights $/ \mathrm{s}(1.9 \mathrm{~m} / \mathrm{s})$.

Two classes of stimuli were generated, one for each of two conditions. The first class had only display motions corresponding to optical flow; the second had these motions plus rotational motions corresponding to the bounce and sway stimuli of Experiment 3 (and Equation 12). That is, the center of display (and everything else in the image) moved sinusoidally up and down through 20 pixels, cycling roughly every $460 \mathrm{~ms}$, and it moved horizontally through 14 pixels, cycling roughly every $920 \mathrm{~ms}$.

Procedures followed were like those of Experiments 4 and 5 . Participants waited for a probe on the last frame of the trial that stayed on the screen until they determined whether it was to the left or the right of the aimpoint. Each condition consisted of a random sequence of 80 trials: 5 probe-aimpoint angles $\left(0.5^{\circ}, 1^{\circ}, 2^{\circ}, 4^{\circ}\right.$, and $\left.8^{\circ}\right) \times 2$ probe directions (left or right of the aimpoint) $\times 4$ aimpoints $\left( \pm 1\right.$ and $3^{\circ}$, as in Warren \& Hannon, 1990, Experiments 1 \& 2) $\times$ replications. The 12 viewers from Experiment 3 participated. Half of the participants viewed stimuli in the pure optical motion condition before those in the condition with rotational eye movements added; half viewed these stimuli in the reverse order.

\section{Results and Discussion}

Results are shown in Figure 16. Performance in the pure optical flow condition was superior to that of the condition with retinal rotations added, $F(1,11)=20.9, p<.001$. Median $75 \%$ and $95 \%$ performance levels were $0.5^{\circ}$ and $2.1^{\circ}$ for optical flow stimuli and $1.1^{\circ}$ and $4.2^{\circ}$ for those with retinal flows added. The increase in the thresholds is considerably greater than the $0.2^{\circ}$ of oscillating motions. From our experience with similar displays, it is clear that larger rotations would impede performance more. In fact, we chose relatively small rotations, in part because larger ones tend to make viewers sick.

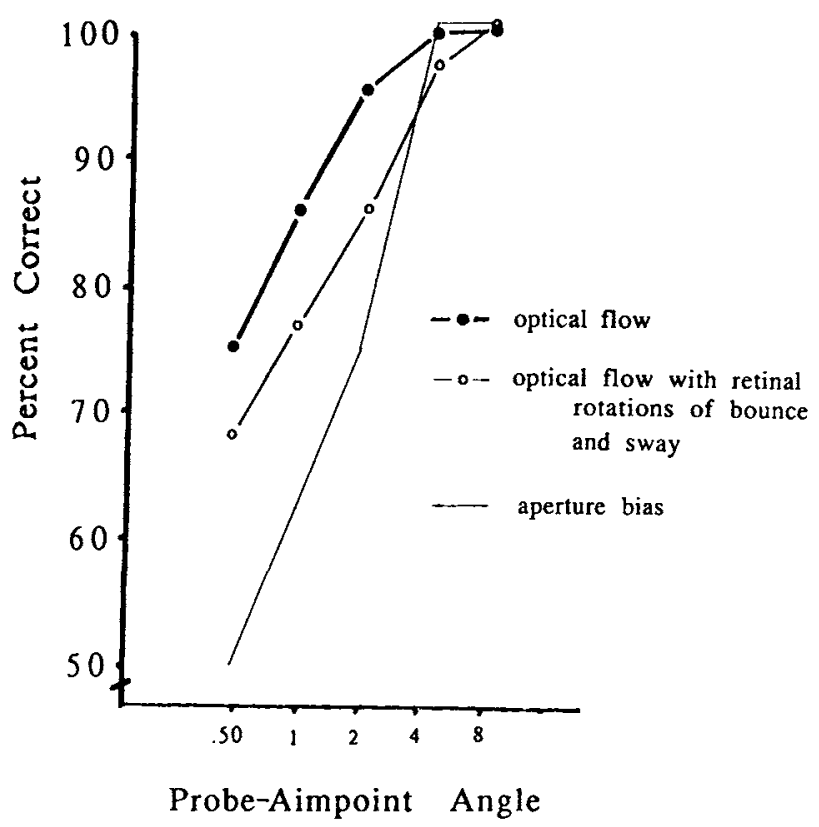

Figure 16. Results of Experiment 7 for wayfinding performance from optical flow with and without the flow due to counterrotation of the eye during bounce and sway of natural gait. (These rotations are less than $0.20^{\circ}$, but double threshold performance. A function based on a response bias to the position of the probe is again superimposed.)

Biases were also present in the data. Performance, shown as if it were based purely on bias, is indicated as the third function in Figure 16. Partial correlations across subjects for all 40 trial types showed reliable effects of probe-aimpoint direction $(r s=.86$ and $.64, p<.0001)$ and bias $(r=.15, p<$ .02 , and $r=.40, p<.0001$ ) for the optical flow and optical flow plus retinal rotation conditions, respectively.

These results resemble those of Simpson (1988), who found that the addition of rotational flow to optical flow considerably increased threshold judgments in the perception of timeto-contact. We conclude that the space-time aliasing in the presentation of low-velocity elements on raster-scan systems (even with relatively high resolution) presents the observer with an artifact that can aid his or her performance in a laboratory abstraction of a wayfinding task.

\section{General Discussion}

The results of Experiments 4 through 6 replicated the overall findings of W. H. Warren et al. (1988) in exquisite detail, but two disturbing problems arose. The first concerns a perceptual bias to see the aimpoint in an optical flow field in the center of the display. The second and more serious problem was pixel creep, the space-time aliasing in raster-scan displays. This information would allow the observer to determine the aimpoint on the basis of an artifact-the interpolated rates of lateral, stepwise movements of several disks across the display. Experiment 7 confirmed the use of this artifact as a viewer aid in performing the task; that is, when 
the small rotational flow fields of eye movements of bounce and sway were added to optical flow, performance was significantly impeded.

Thus, Experiments 1 through 3 raise many issues in support of DMP for wayfinding, and artifacts in Experiments 4 through 7 raise some issues against IOF. It remains logically possible, however, that those experiments supporting DMP and those supporting IOF are simply measuring different aspects of the same thing. After all, as DMP is a phenomenon of retinal flow, and as IOF is a component of retinal flow, perhaps the two potential sources of information are closer than these experiments and this presentation would allow. If so, experiments on optical and retinal flow ought to yield parallel results in all circumstances. Experiments 8 and 9 dissociate the two sources of information.

\section{Dissociations of Information Use From Retinal and Optical Flow Fields}

Our final two studies focus on a double dissociation of IOF and DMP; that is, we found one manipulation that drastically affected performance with optical flow displays but not retinal flow displays and another manipulation that created the reverse effect. More concretely, Experiment 8 reconsiders the decomposition process, taxing the visual system more heavily than in Experiment 7 with exaggerated motions of bounce and sway, and Experiment 9 uses unnatural displays in pursuing the role of rigidity in IOF and DMP.

\section{Experiment 8: Taxing Decompositional and Nondecompositional Processes}

It might be argued that the results of Experiment 7 were inconclusive. To be sure, there was a reliable decrement in performance to IOF when small rotational oscillations were added to the optical flow field, but that decrement was relatively small, and perhaps unimportant. Moreover, because Experiments 1 through 3 typically found small (sometimes significant, sometimes not) decrements in performance for DMP with the addition of bounce and sway motions to retinal flow, the conclusions concerning the disruption of decompositional processes might be overstated.

What is needed is a more direct and taxing comparison between optical and retinal information under conditions of bounce and sway. This was accomplished by exaggerating the amount of bounce and sway optical and retinal flows in Equations 11 and 12 . In each case, the magnitudes were five times greater than those used in Experiments 2 and 7.

\section{Method}

Four classes of stimuli were generated, two concerning IOF and decomposition and two concerning DMP.

The first condition used pure optical flow identical to that in Experiment 7 . In the second condition, optical flow was compounded with retinal rotational motions that were five times greater than those in Experiment 7, but still followed Equation 12. That is, in the latter stimuli the rotational oscillations moved the trees in the stimuli vertically through \pm 50 pixels every $460 \mathrm{~ms}\left( \pm 1^{\circ}\right)$ and horizontally through \pm 30 pixels every $920 \mathrm{~ms}\left( \pm 0.7^{\circ}\right)$. Stimulus durations, frame rates, tree populations, and forward velocities were the same as in Experiment 7. Each condition consisted of 80 trials: 5 probe-aimpoint angles $\left(0.5^{\circ}, 1^{\circ}, 2^{\circ}, 4^{\circ}\right.$, and $\left.8^{\circ}\right) \times 2$ probe directions (left and right of the aimpoint) $\times 2$ aimpoints $\left( \pm 3^{\circ}\right.$ left or right of screen center $) \times 4$ replications.

Retinal flow conditions were yoked in structure to the optical flow conditions. Thus, there were again 80 trials in each condition: 5 gazemovement angles $\left(0.5^{\circ}, 1^{\circ}, 2^{\circ}, 4^{\circ}\right.$, and $\left.8^{\circ}\right) \times 2$ directions of movement (to the left and right of fixation) $\times 8$ replications. In one condition the stimuli simulated only smooth dolly and pan motions, and in the other the very large bounce and sway motions were superimposed. Retinal rotations were exactly the same as those used when superimposed on the optical flow condition; optical counterrotations (due to simulated change in eye height and lateral position) were synchronized to these, as in all previous DMP experiments presented here.

James E. Cutting, Paul A. Braren, and Scott H. Johnson each participated in three sessions, yielding 240 trials per condition. This somewhat unusual procedure is not ideal, but it was followed because, as noted in the discussion of the results of Experiment 7, the large oscillations tend to make viewers queasy; they are not particularly easy to sit through. Because these displays had the potential to make participants sick, we were unwilling to subject naive viewers to them.

\section{Results}

Results of the four conditions are shown in the two panels of Figure 17. As usual there was a large effect of angle (either probe-aimpoint or gaze-movement), $F(4,8)=185.9, M S_{e}=$ $185.3, p<.0001$. There was also a reliable effect of carriage, $\left.F(1,2)=27.1, M S_{\mathrm{e}}=86.8, p<.035\right)$; that is, performance in the pure optical and pure retinal flow conditions was superior to performance in the corresponding conditions with additional exaggerated flow patterns. This result is not surprising as the exaggerated conditions are the ones that come close to inducing motion sickness. Performance in the two optical and two retinal flow conditions, however, did not differ, $F(1,2)=5.58, p>.14$. Most important, however, there was an Angle $\times$ Carriage $\times$ Flow condition interaction, $F(4$, $8)=5.30, M S_{\mathrm{e}}=5.02, p<.025$. In particular, performance in the optical flow plus exaggerated rotations condition was worse at small angles than in any other condition.

Inspecting performance in the four conditions in a pairwise fashion showed no difference between the pure optical and pure retinal flow conditions, $F(1,2)=0.37, p>.60$, but reliable differences were found between the two optical flow conditions at different angles (right panel of Figure 17), $F(4$, $8)=7.3, M S_{\mathrm{e}}=18.1, p<.01$, and between the two retinal flow conditions overall (left panel of Figure 17), $F(1,2)=$ 88.9, $M S_{\mathrm{e}}=12.8, p<.02$. Most important, however, comparing the two conditions with exaggerated rotations added, performance in the retinal flow condition was superior to that in the optical flow condition at small angles, $F(4,8)=6.22$, $M S_{\mathrm{e}}=6.58, p<.015$. We take this latter result as our strongest evidence against decomposition. That is, comparing Equations 11 and 12 shows fewer component flow fields in the optical condition than in the retinal condition, and yet performance was worse in the former.

Moreover, we think the difference between the two conditions with exaggerated motions is underestimated. Performance in the optical flow condition with rotations was as good as it was in large part because of the response bias. 
OPTICAL FLOW

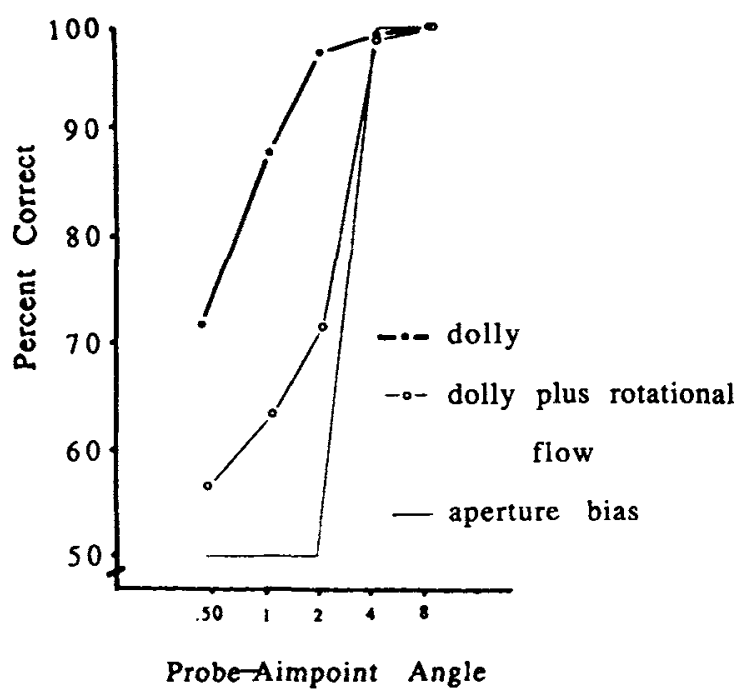

RETINAL FLOW

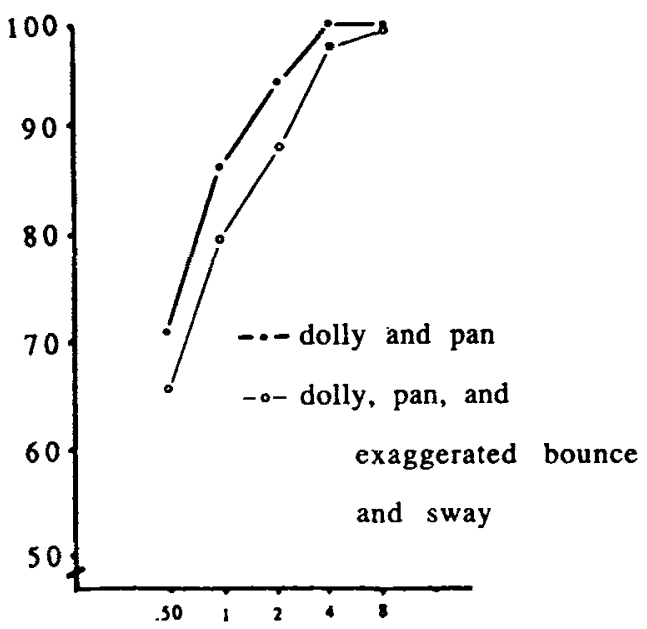

Final Gaze-Movement Angle

Figure 17. Results of Experiment 8 for wayfinding performance to optical and retinal flows. (Optical flows occurred with and without large oscillations and retinal flows occurred with and without similarly large amounts of bounce and sway. The optical flow conditions assess the feasibility of decomposition as presented in Equations 4 and 12. The retinal flow conditions assess nondecomposition in Equations 4 and 11 . Superimposed on the optical flow conditions is a response bias function, as in previous figures.)

Performance based on bias alone is plotted as the third function in the left panel of Figure 17. Partial correlations showed the bias to be superior in strength to the probeaimpoint direction $(r s=.73$ and .43 , respectively, both $p s<$ .0001 . The bias was not nearly as strong in the pure optical flow condition $(r=.06, p<.05)$ and probe-aimpoint direction was stronger $(r=.96, p<.0001)$.

In summary, Experiment 8 shows our first dissociation between IOF and DMP. IOF in a situation with fewer oscillating flow fields seems more difficult to extract than DMP with more oscillating flow fields. In Experiment 9, we investigated another dissociation.

\section{Experiment 9: Wayfinding From Retinal and Optical Flow Fields in Rigid and Nonrigid Environments}

The original formulations of wayfinding from optical flow (J. J. Gibson, 1947, 1950; Calvert, 1950) have a curious property. That is, they do not assume that the observer is traveling through a rigid environment. Instead, all that is required is that the directions of all vectors in the optical flow field are oriented such that they point away from the aimpoint. Vector magnitudes are irrelevant; they are information about depth. In contrast, more contemporary treatments of optical flow (Koenderink \& van Doorn, 1981; LonguetHiggins \& Prazdny, 1980) emphasize rigidity, and W. H. Warren et al. (1988), for example, stated, "the radial flow pattern is independent of the distances and shapes of environmental surfaces and specifies heading in any rigid [italics added] environment, requiring no assumption about surface shape or smoothness" (pp. 647-648). However, rigidity need not be assumed to obtain a radial flow field for linear translation.

What kind of nonrigid environments preserve radial vectors in optical flow? The general class is suggested in the left panel of Figure 18. If an observer moves on a linear path, and if a random-lengthed vector parallel to the observer's path is added to all objects on a ground plane, an optical flow field with anomalous vector lengths is obtained. If the additional random vectors are small, this environment can be interpreted as nonplanar, like a cloud of objects at different eye heights (e.g., Warren \& Hannon, 1990; Warren et al., 1991). However, if the random vectors are of sufficient magnitude to reverse the flow (moving toward the aimpoint), no rigid environment can accommodate these motions; it must be nonrigid.

It seems possible that observers could estimate aimpoints in certain nonrigid environments about as well as in rigid ones. If so, such data would go some distance toward impugning theories of wayfinding based on decomposition and optical flow. ${ }^{22}$ That is, the data would be decoupled from the theory

\footnotetext{
${ }^{22}$ Although there are many computational models of decomposition, in general they fall into two classes: those using determinate solutions (e.g., Longuet-Higgins \& Prazdny, 1980; Waxman \& Ullman, 1985) and those using error-minimization methods (e.g., Bruss \& Horn, 1983; Prazdny, 1981). The determinate solutions often use spatial derivatives of flow, and thus assume rigidity; the minimization methods, on the other hand, are likely to fare better in nonrigid environments. They often compute flow lines, which regardless of length would align themselves to the aimpoint.
} 


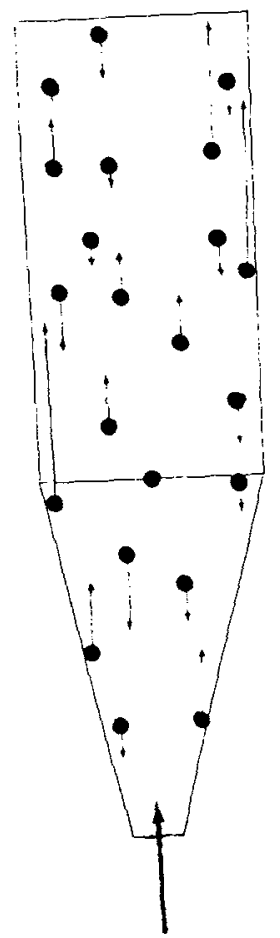

OPTICAL FLOW

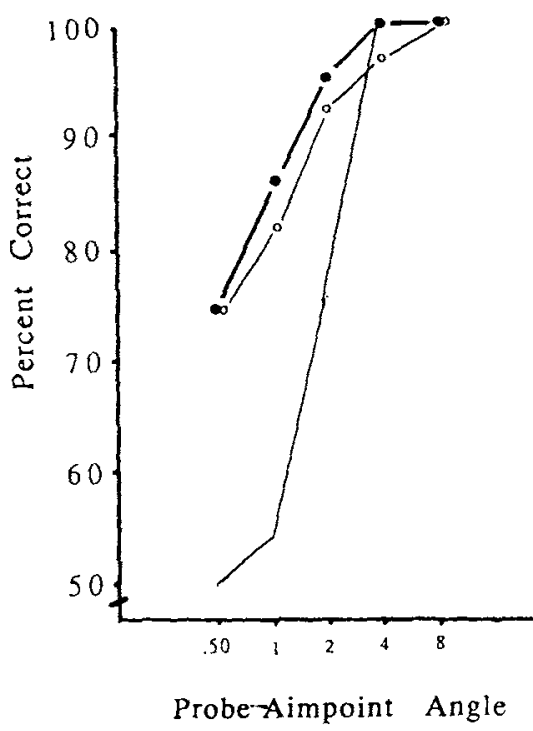

RETINAL FLOW

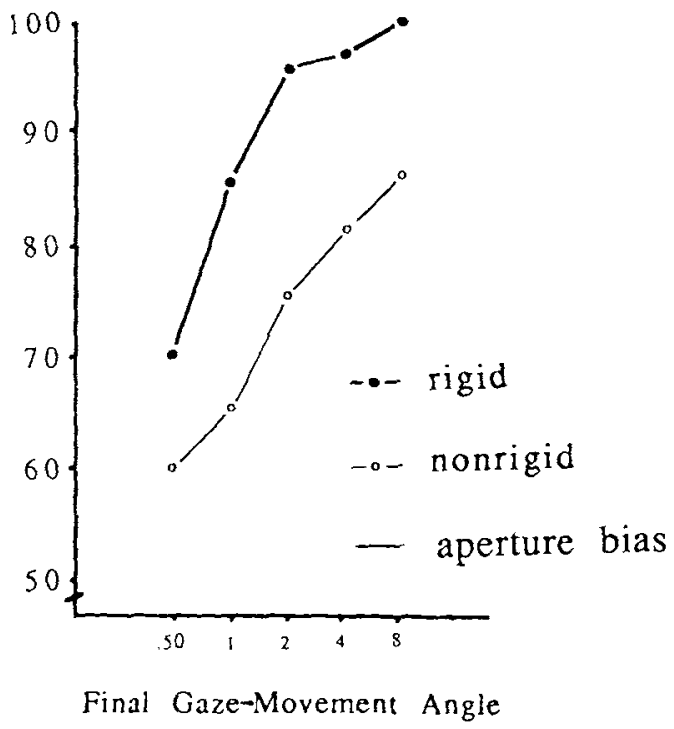

Figure 18. The environment and the results of Experiment 9. (The left panel represents a bird's-eye view of a nonrigid environment that preserves the radial nature of information in optical flow [IOF] but will generally destroy the trustworthiness of differential motion parallax [DMP]. Because vector length is near random in the optical flow, no consistent depth pattern could be computed. The middle and right panels show results comparing DMP and IOF in rigid and nonrigid environments. The data for the rigid optical flow condition are the same as in Figure 16. The response bias function is again superimposed. The interaction of flow and rigidity demonstrates the psychological nonidentity of DMP and IOF.)

because they apply to a domain broader than the theory allows. This would underline the necessity of a new theory that accounts for all the data.

DMP, on the other hand, is very much tied to a rigidity assumption; a moving observer is not likely to be able to find his or her way when relative retinal motions are inconsistent with a rigidity assumption. ${ }^{23}$ Thus, the predicted dissociation is this: Waytinding performances ought to be the same in rigid and certain nonrigid environments based on optical flow, but performances ought to be quite different in these environments based on retinal flow.

\section{Method}

Stimuli were again like those of Experiment 7, consisting of a mean of 21 trees. Again frames were $120 \mathrm{~ms}$ each and trial durations were $4.3 \mathrm{~s}$; and again subjects could not terminate the trials. This time, however, no stimuli had bounce and sway motions of any kind.

There were four conditions: two for optical flow (fixed-cameraangle setting) and two for retinal flow (simulated pursuit fixation); also, two conditions had rigid environments and two had nonrigid environments. The optical flow conditions were the same as those in Experiments 4 and 5 , which were produced using a fixed-cameraangle technique and a postmotion probe. (The methods and the data from the condition with a rigid environment and optical flow have already been reported as the pure optical flow condition in Experiment 7.) The two retinal flow conditions were the same as those in Experiment 1, which were produced using a simulated pursuitfixation technique with no probe. Viewers simply waited until the end of the trial to give a response indicating whether they were looking to the right or left of their path of movement. In optical flow conditions there were 80 trials, as noted in Experiment 7; in the retinal flow conditions there were also 80 trials: 5 final gaze-movement angles $\left(0.5^{\circ}, 1^{\circ}, 2^{\circ}, 4^{\circ}\right.$, and $\left.8^{\circ}\right) \times 2$ gaze directions (left and right) $\times 8$ replications. All trials were randomized within conditions.

In the two optical flow conditions, the locations of the trees were random with no restrictions. In the two retinal flow conditions, the fixation tree was always at center screen (and colored black), at a distance of 15.6 eye heights, with the other objects randomly placed. The horizon was at 62.5 eye heights in all conditions. Rigid environ-

${ }^{23}$ DMP may still work in certain kinds of nonrigid environments. For example, the stochastic and regular deviations from rigidity seen on the surface of a lake, or in the branches moving in a tree over time, may still offer sufficient DMP information for wayfinding. Neither of these types of nonrigidity, however, is similar to that used in Experiment 9. The force of Experiment 9 is to show that DMP and IOF can be dissociated with a particular kind of nonrigid environment, not that they would be dissociated in all kinds of nonrigid environments. 
ments were like those in Experiments 1 through 7, and simulated forward velocity was 1.2 eye heights $/ \mathrm{s}(1.9 \mathrm{~m} / \mathrm{s})$. In nonrigid environments, however, the trees were given an additional $z$-axis motion vector along the ground plane, parallel to the observer's linear path. Magnitudes of these motion vectors were randomly chosen between values of $1.9 \mathrm{~m} / \mathrm{s}$ and $-3.8 \mathrm{~m} / \mathrm{s}$. Thus, separate velocities for each object corresponded to forward movement of the observer within the range of $3.8 \mathrm{~m} / \mathrm{s}$ to $-1.9 \mathrm{~m} / \mathrm{s}$, with a mean of $0.95 \mathrm{~m} / \mathrm{s}$. Negative numbers correspond to the observer moving backward. Obviously, because the observer cannot travel simultaneously at a range of speeds, the arrangement of the trees was nonrigid. These nonrigid stimuli looked odd, even eerie, like so many trees purposefully "commuting" to and from the horizon, but generally following unknown traffic laws and paying "no attention" to other trees.

The same 12 observers as those in Experiments 3 and 7 participated, but they participated in different parts of the experiment first. ${ }^{24}$ Half participated in all three optical flow conditions (Experiments 7 and 9) before the three retinal flow conditions (Experiments 3 and 9 ), and half participated in reverse order. Order was fixed within optical and retinal flow conditions. Thus, 6 subjects viewed (a) the rigid environment, optical flow condition of Experiment 9 (and Experiment 7) first; (b) the condition of Experiment 7 with optical flow plus two rotational flow fields; (c) the nonrigid, optical flow condition of Experiment 9; (d) the rigid, retinal flow condition of Experiment 9; (e) Experiment 3; and then (f) the nonrigid, retinal flow condition of Experiment 9. The other 6 subjects viewed the conditions in the following order: (d), (e), (f), (b), (a), and (c).

\section{Results and Discussion}

Results are shown in the right two panels of Figure 18. As expected, there was a large effect of angle (either gaze-movement or probe-aimpoint), $F(4,44)=73.4, M S_{e}=137.6, p<$ .0001 . There was also a main effect of flow field, with optical motion displays superior to retinal motion displays, $F(1,11)$ $=20.6, p<.001$, and a main effect of rigidity, with rigid displays superior to nonrigid ones, $F(1,11)=34.1, p<.001$.

However, the latter two main effects were due to an interaction between rigidity and flow field, $F(1,11)=20.58, M S_{\mathrm{e}}$ $=121.8, p<.001$. That is, performance for nonrigid, retinal stimuli was systematically worse than performance for stimuli in the other three conditions. There was no reliable difference between the rigid and nonrigid optical flow conditions, $F(1$, 11) $\left.=1.7, M S_{\mathrm{e}}=4.8, p>.20\right)$ and no reliable difference between the rigid optical and rigid retinal flow conditions, $F(1,11)=2.49, M S_{\mathrm{e}}=4.4, p>.14$.

The interaction between rigidity and flow field demonstrates a strong dissociation between information in optical and retinal flows. Thus, DMP and IOF cannot be variants of the same source of information. The superiority of IOF to DMP in nonrigid environments should not be taken as evidence for the superiority of IOF; no terrestrial animal evolved in a nonrigid environment.

\section{General Discussion}

Two results are most important across Experiments 8 and 9. First, the stimulus manipulations used in these two experiments successfully dissociated observer performance on tasks offering IOF and DMP to observers. In particular, when large rotational motions were added to IOF and DMP, performance was impeded more for IOF displays, and when a certain class of environmental nonrigidity underlay IOF and DMP, performance was impeded more for DMP displays. Thus, DMP and IOF cannot be notational variants of the same information.

Second, in Experiment 8, performance was significantly worse when large rotational motions were added to the IOF displays. This performance decrement was much larger than when identical rotational motions (and compensatory optical motions) were added to DMP displays. This pattern of results has strong implications against decomposition.

\section{Against Decomposition and the Use of Optical Flow for the Pedestrian}

We think the imperative of the logic and data from Experiments 4 through 9 speaks against decomposition of retinal flow and against the use of optical flow at pedestrian speeds. Five considerations bring us to these conclusions.

\section{Three Arguments Against Decomposition}

First, and harkening back to our original arguments, we appeal to parsimony. If DMP in retinal flow suffices, why carry on with the process of decomposition? Decomposition could only verify a solution already obtainable by other, previous means.

Second, the retinal flow fields for stimuli emulating natural gait, with full bounce and sway, have six components, as shown in Equation 11. Five of these flow fields are irrelevant to wayfinding. Decomposition schemes would seem overtaxed by this swirling plethora of irrelevancy. Surely, if decomposition is a procedure that can be taxed, one would predict inferior performance on trials with bounce and sway as compared with those without. No evidence of this was found in Experiments 2 and 3, however, and little, if any, was found in Experiment 1 for tasks involving simulated pursuit fixation. Only in Experiment 8, with five times the normal amount of bounce and sway, were decrements to performance found. These results seem due to the potential of bounce and sway for inducing motion sickness and not to their lack of informativeness.

Third, when displays with three component flow fieldstwo retinal and one optical-were combined in Experiment 7 , performance was impeded when compared with displays with only optical flow. In Experiment 8, this performance difference was even greater. This degradation could only arise

\footnotetext{
${ }^{24}$ A total of 14 subjects participated, but 2 were dropped because they did not follow directions on the DMP tasks. Their confusion arose from a response incompatibility across the IOF and DMP tasks. In IOF tasks, observers indicated whether the probe was to the left or right of their path; in DMP tasks (consistent with previous studies), they indicated whether their path was to the left or right of the fixation tree. Both subjects who were dropped had problems because their DMP tasks followed their IOF tasks, and they became confused part way through the DMP task, switching their responses. In general, the instructions for IOF tasks are more easily given and more easily understood.
} 
as a difficulty in decomposing the retinal flow field to arrive at IOF. Because no such difficulty typically arises in compounded flow fields for DMP, decomposition (if it occurs) seems insufficiently successful for wayfinding tasks.

\section{Two Arguments Against the Use of Optical Flow}

First, and again harkening back to earlier arguments, consider again the optical flow pattern itself, as shown in Figure 2 . In the computed flow pattern, the vector lengths within about $10^{\circ}$ of the aimpoint are very small for movement across a flat terrain, and they are not large at pedestrian speeds within $15^{\circ}$. To use optical flow in such situations, an observer must interpolate large distances across the visual field to find the aimpoint.

Second, in some studies pixel creep seems to augment, if not substitute for, IOF in wayfinding judgments from optical flow. Thus, viewers may not be using IOF in those studies at all, because superior performance is attainable through the use of a display artifact.

\section{Velocity Effects, Retinal Flow, and Natural Gaze Patterns}

Although we claim that DMP is used for wayfinding at pedestrian speeds, we hasten to add that as an individual moves faster and faster through the environment, the situation will change. It changes because eye movement and head movement behavior is yoked to flow velocities. As suggested by the quote from Schivelbusch (1986) that we used to set the stage for DMP, it is difficult to fixate and pursue a nearby object when one is moving fairly fast. Calvert (1954), for example, noted

\footnotetext{
When a cinematographic film is taken of the face of a driver of a vehicle starting from rest, it is found that he scans the visual field only when the vehicle is moving slowly. As the speed increases, the driver scans less and less, until finally he begins to stare fixedly in the direction in which he wishes to go, usually at his aiming point if he can see it, or at some point close to it if he is unable to see it. The higher the speed and the more difficult the task, the stronger the tendency to fixate, and the less likely the pilot is to glance at objects far removed from his path. (p. 240)
}

Thus, as one's speed increases, fewer eye and head movements are made off path until a velocity is reached at which the eyes are riveted to the aimpoint. When this occurs, optical and retinal flow fields are the same because the eye undergoes no rotation (see Equation 4).

When traveling in a train at greater than 10 eye heights/s, observers cannot use DMP unless they look out far enough into the distance to fixate an object. Nearby objects whiz by. Indeed, high-speed observers prefer not to look to the side, and if they can they will look to the front. If they look near their aimpoint, a radial pattern in the retinal array can be used to guide their movements; if they look somewhat off to the side, the curvature in their retinal flow field may also prove useful (see Warren et al., 1990, for the optical analysis). Thus, we claim that retinal information is used for wayfinding throughout, but that a shift is made from DMP to global radial pattern when either eye movements become too fatiguing or pursuit fixations are no longer trustworthy.

\section{Conclusion}

We conclude that moving observers avoid objects, plan their routes, and generally find their way through cluttered environments on the basis of information in retinal flow. At pedestrian speeds, that information seems largely contained in DMP, although other sources related to DMP may be available and may be used as well at different velocities and in different situations with varying degrees of clutter.

We reject the idea that observers use IOF on four grounds. First, DMP provides an adequate account of both correct and incorrect performance in wayfinding tasks (Experiments 1 and 2), whereas current accounts of IOF do not. Second, the decomposition procedure of going from retinal to optical flow fields is unnecessary, because adequate information is available in the DMP of retinal flow (Experiments 2 and 3), necessarily before decomposition. Third, the addition of other rotational or optical flow components to a stimulus does not generally impede performance in wayfinding experiments based on DMP (Experiments 1, 2, and 3, but see also Experiment 8 ), but does impede performance where IOF is used (Experiments 7 and 8 ). Fourth, artifacts in the current experiments are thought to reflect the use of IOF (as shown in Experiments 4 through 7 ). We also reject the idea that there is no principled difference between uses of retinal and optical flow. Experiments 8 and 9 showed a double dissociation between the information in the two types of flow fields that countermands any argument for their similarity.

Although optical flow is an elegant mathematical concept, it may be that for creatures with mobile eyes and foveas (like human beings) it has no psychological reality. We think those who have endorsed the use of optical flow have made a deep mistake; in searching for information (in optical flow) that is putatively closer to the ecology of observers moving through their environments, they have ignored the proximal information (in retinal flow) presented to the observers' senses. Those recognizing this error created the scheme of decomposition (e.g., Longuet-Higgins \& Prazdny, 1980), a patchwork process without empirical support. On the basis of our results, we think decomposition is a nonexistent psychological process intended to link the ecological fiction of optical flow to real retinal information. For the purpose of wayfinding, we prefer our analysis of the retinal information: it works, it is simpler, and it accounts for the data.

\section{References}

Ahumada, A. J. (1983). Bias and discriminability in aimpoint estimation. Unpublished manuscript, National Aeronautics and Space Administration Ames Research Center, Moffett Field, CA.

Allman, J., Miezin, F., \& McGuiness, E. (1985). Direction- and velocity-specific responses from beyond the classical receptive field in the middle temporal visual area (MT). Perception, 14, 105-126. Andersen, G. J. (1990). Segregation of optic flow into object and self- 
motion components: Foundations for a general model. In R. Warren \& A. H. Wertheim (Eds.), Perception and control of self-motion (pp. 127-142). Hillsdale, NJ: Erlbaum.

Andersen, G. J., \& Braunstein, M. (1985). Induced self-motion in central vision. Journal of Experimental Psychology: Human Perception and Performance, 11, 122-132.

Berkeley, G. (1937). An essay towards a new theory of vision. In The works of George Berkeley (pp. 86-116). London: Thomas Tegg \& Son. (Original work published 1703).

Bridgeman, B. (1972). Visual receptive fields sensitive to absolute and relative motion during tracking. Science, 173, 1106-1 108.

Bruss, A. R., \& Horn, B. K. P. (1983). Passive navigation. Computer Vision, Graphics, and Image Processing, 21, 3-20.

Burton, G., \& Turvey, M. T. (1990). Perceiving the lengths of rods that are held but not wielded. Ecological Psychology, 2, 295-324.

Calvert, E. S. (1950). Visual aids for landing in bad visibility, with particular reference to the transition from instrument to visual flight. Transactions of the Illuminating Engineering Society, London, 15, 3-20.

Calvert, E. S. (1954). Visual judgments in motion. Journal of the Institute of Navigation, London, 7, 233-251.

Carel, W. L. (1961). Visual factors in the contact analog. (Publication R61 ELC60, pp. 1-65). Ithaca, NY: General Electric Advanced Electronics Center.

Carlsöö, S. (1972). How man moves. London: Heinemann.

Clocksin, W. F. (1980). Perception of surface slant and edge labels from optical flow: A computational analysis. Perception, 9, 253267.

Cutting, J. E. (1983). Perceiving and recovering structure from events. In Proceedings of the SIGGRAPH/SIGART Interdisciplinary Workshop (pp. 141-147). New York: Association for Computing Machinery.

Cutting, J. E. (1986). Perception with an eye for motion. Cambridge, MA: MIT Press.

Cutting, J. E. (1991a). Four ways to reject directed perception. Ecological Psychology, 3, 25-34.

Cutting, J. E. (1991b). Why our stimuli look as they do. In G. Lockhead \& J. R. Pomerantz (Eds.), Perception of structure: Essays in honor of Wendell R. Garner (pp. 41-52). Washington, DC: American Psychological Association.

Cutting, J. E., Proffitt, D. R., \& Kozlowski, L. T. (1978). A biomechanical invariant for gait perception. Journal of Experimental Psychology: Human Perception and Performance, 4, 357-372.

Dichgans, J., \& Brandt, T. (1978). Visual-vestibular interactions and motion perception. In R. Held, H. W. Leibowitz, \& H. L. Teuber (Eds.), Handbook of sensory physiology (Vol. 8, pp. 755-804). Berlin, Federal Republic of Germany: Springer-Verlag.

Duncker, K. (1938). Induced motion. In W. D. Ellis (Ed. and Trans.), A source book of Gestalt psychology (pp. 161-172). New York: Humanities Press. (Original work published 1912).

Frost, B. J., \& Nakayama, K. (1983). Single visual neurons code opposing motion independent of direction. Science, 220, 774-775.

Gibson, E. J., Gibson, J. J., Smith, O. W., \& Flock, H. (1959). Motion parallax as a determinant of perceived depth. Journal of Experimental Psychology, 58, 40-51.

Gibson, J. J. (Ed.). (1947). Motion picture testing and research (AAF Aviation Psychology Research Report No. 7). Washington, DC: U. S. Government Printing Office.

Gibson, J. J. (1950). The perception of the visual world. Boston: Houghton Mifflin.

Gibson, J. J. (1966). The senses considered as perceptual systems. Boston: Houghton Mifflin.

Gibson, J. J. (1979). The ecological approach to visual perception. Boston: Houghton Mifflin.

Gibson, J. J., Olum, P., \& Rosenblatt, F. (1955). Parallax and perspective during aircraft landings. American Journal of Psychology, 58, 372-385.

Gogel, W. (1982). Analysis of the perception of motion concomitant with a lateral motion of the head. Perception \& Psychophysics, 32, $241-250$.

Gordon, D. A. (1965). Static and dynamic visual fields in human space perception. Journal of the Optical Society of America, 55, 1296-1303.

Hasbrook, A. H. (1975, November). The approach and landing. Business and Commercial Aviation, 39-43.

Helmholtz, H. von (1925). Treatise on physiological optics (J. Southall, Trans.). Menosha, WI: Optical Society of America.

Hochberg, J. (1986). Representation of motion and space in video and cinematic displays. In K. R. Boff, L. Kaufman, \& J. P. Thomas (Eds.), Handbook of perception and human performance (Vol. 1, Chap. 22:1-64). New York: Wiley.

Johansson, G. (1977). Studies on the visual perception of locomotion. Perception, 6, 365-376.

Johnston, I. R., White, G. R., \& Cumming, R. W. (1973). The role of optical expansion patterns in locomotor control. American Journal of Psychology, 86, 311-324.

Judge, S. J. (1990). Knowing where you're going. Nature, 348, 115.

Kaufman, L. (1968). Research in visual perception for carrier landing (Suppl. 2). Great Neck, NY: Sperry Rand Research Center.

Koenderink, J. J. (1985). Space, form, and optical deformations. In D. Ingle, M. Jeannerod, \& D. N. Lee (Eds.), Brain mechanisms and spatial vision (pp. 31-58). Dordrecht, The Netherlands: Martinus Nijhoff.

Koenderink, J. J. (1986). Optic flow. Vision Research, 26, 161-180.

Koenderink, J. J. (1990). Some theoretical aspects of optic flow. In R. Warren \& A. H. Wertheim (Eds.), Perception \& control of selfmotion (pp. 53-68). Hillsdale, NJ: Erlbaum.

Koenderink, J. J., \& van Doorn, A. J. (1981). Exterospecific component for the detection of structure and motion in three dimensions. Journal of the Optical Society of America, 71, 953-957.

Koenderink, J. J., \& van Doorn, A. J. (1987). Facts on optic flow. Biological Cybernetics, 56, 247-254.

Langewiesche, W. (1944). Stick and rudder. New York: McGrawHill.

Larish, J. F., \& Flach, J. M. (1990). Sources of optical information useful for perception of speed of rectilinear self-motion. Journal of Experimental Psychology: Human Perception and Performance, 16 , 295-302.

Lee, D. N. (1980). The optic flow field. Philosophical Transactions of the Royal Society, London, B, 290, 169-179.

Lee, D. N., \& Lishman, R. (1977). Visual control of locomotion. Scandinavian Journal of Psychology, 18, 224-230.

Lee, D. N., Lishman, R., \& Thomson, J. A. (1982). Regulation of gait in long jumping. Journal of Experimental Psychology: Human Perception and Performance, 8, 448-459.

Llewellyn, K. R. (1971). Visual guidance of locomotion. Journal of Experimental Psychology, 91, 245-261.

Longuet-Higgins, H. C., \& Prazdny, K. (1980). The interpretation of a moving retinal image. Proceedings of the Royal Society, London, $B, 208,385-297$.

Macgowan, K. (1965). Behind the screen. New York: Delacorte Press. Molyneux, W. (1690). Dioptrica nova. London: Benjamin Tooke.

Murray, M. P. (1967). Gait as a total pattern of movement. American Journal of Physical Medicine, 46, 290-333.

Nakayama, K. (1985). Extraction of higher order derivatives of the optical velocity vector field: Limitations imposed by biological hardware. In D. Ingle, M. Jeannerod, \& D. N. Lee (Eds.), Brain mechanisms and spatial vision (pp. 59-71). Dordrecht, The Netherlands: Martinus Nijhoff.

Nakayama, K., \& Loomis, J. M. (1974). Optical velocity patterns, 
velocity sensitive neurons, and space perception: A hypothesis. Perception, 3, 63-80.

Owen, D. H. (1990). Lexicon of terms of the perception and control of self-motion and orientation. In R. Warren \& A. H. Wertheim (Eds.), Perception \& control of self-motion (pp. 33-50). Hillsdale, NJ: Erlbaum.

Patla, A. E., Prentice, S. D., Robinson, C., \& Neufield, D. (1991). Visual control of locomotion: Strategies for changing direction and for going over obstacles. Journal of Experimental Psychology: $\mathrm{Hu}$ man Perception and Performance, 17, 603-634.

Perrett, D., Harries, M., Mistlin, A. J., \& Chitty, A. J. (1990). Three stages in the classification of body movements by visual neurons. In H. Barlow, C. Blakemore, \& M. Weston-Smith (Eds.), Images and understanding (pp. 94-107). Cambridge, England: Cambridge University Press.

Perrone, J. A. (1980). Slant underestimation: A model based on the size of the viewing aperture. Perception, 9, 285-302.

Prazdny, K. (1981). Determining the instantaneous direction of motion from optical flow generated by a curvilinearly moving observer. Computer Graphics and Image Processing, 17, 238-248.

Prazdny, K. (1983). On the information in optic flows. Computer Vision, Graphics, and Image Processing, 22, 235-259.

Priest, H., \& Cutting, J. E. (1985). Visual flow and direction of locomotion. Science, 277, 1063-1064.

Probst, T., Krafczyk, S., Brandt, T., \& Wist, E. R. (1984). Interaction between perceived self-motion and object-motion impairs vehicle guidance. Science, 225, 536-538.

Radford, C. J. (1986). Optical flow fields in Hough transform space. Pattern Recognition Letters, 4, 293-303.

Raviv, D. (1990). A quantitative approach to camera fixation (National Institute of Standards and Technology Report 4324. Washington, DC: U. S. Department of Commerce.

Regan, D. M. (1985). Visual how and direction of locomotion. Science, 277, 1064-1065.

Regan, D. M., \& Beverley, K. I. (1978). Looming detectors in the human visual pathway. Vision Research, 18, 415-421.

Regan, D. M., \& Beverley, K. I. (1982). How do we avoid confounding the direction we are looking and the direction we are moving? Science, 215, 194-196.

Regan, D. M., Kaufman, L., \& Lincoln, J. (1986). Motion in depth and visual acceleration. In K. R. Boff, L. Kaufman, \& J. P. Thomas (Eds.), Handbook of perception and human performance (Vol 1., Chap. 19: pp. 1-46). New York: Wiley.

Rieger, J. H. (1983). Information in optical flows induced by curved paths of observation. Journal of the Optical Society of America, A2, $354-560$.

Rieger, J. H., \& Lawton, D. T. (1985). Processing differential image motion. Journal of the Optical Society of America, A, 2, 354-360.

Rieger, J. H., \& Toet, L. (1985). Human visual navigation in the presence of 3-D motions. Biological Cybernetics, 52, 354-360.

Riemersma, J. B. J. (1981). Visual control during straight road driving. Acta Psychologica, 48, 214-225.
Road Research Laboratory. (1963). Research on road safety. London: Her Majesty's Stationery Office.

Rock, I., \& Smith, D. (1986). The optomotor response and induced motion of the self. Perception, 15, 497-502.

Roy, J.-P., \& Wurst, R. H. (1990). The role of disparity-sensitive cortical neurons in signalling the direction of self-motion. Nature, $348,160-162$.

Schivelbusch, W. (1986). The railway journey. Berkeley: University of California Press.

Schivelbusch, W. (1988). The disenchanted night. Berkeley: University of California Press.

Simpson, W. A. (1988). Depth discrimination from optic flow. Perception, 17, 497-512.

Spottiswoode, R. (1968). Film and its techniques. Berkeley: University of California Press.

Stoffregen, T. (1985). Flow structure versus retinal location in the optical control of stance. Journal of Experimental Psychology: Human Perception and Performance, 11, 554-565.

Torrey, C. (1985). Visual flow and direction of locomotion. Science, $277,1064$.

Turvey, M. T. (1990). Coordination. American Psychologist, 45, $938-$ 953.

Warren, R. (1976). The perception of egomotion. Journal of Experimental Psychology: Human Perception and Performance, 2, 448456.

Warren, R. (1990). Preliminary questions for the study of egomotion. In R. Warren \& A. H. Wertheim (Eds.), Perception and control of self-motion (pp. 3-32). Hillsdale, NJ: Erlbaum.

Warren, W. H., Blackwell, A. W., \& Morris, M. W. (1989). Age differences in perceiving the direction of self-motion from optical flow. Journal of Gerontology: Psychological Sciences, 44, P147P153.

Warren, W. H., \& Hannon, D. J. (1988). Direction of self-motion is perceived from optical flow. Nature, 336, 162-163.

Warren, W. H., \& Hannon, D. J. (1990). Eye movements and optical flow. Journal of the Optical Society of America, A, 7, 160-169.

Warren, W. H., Mestre, D. R., Blackwell, A. W., \& Morris, M. W. (1991). Perception of circular heading from optical flow. Journal of Experimental Psychology: Human Perception and Performance, $17,28-43$

Warren, W. H., Morris, M. W., \& Kalish, M. (1988). Perception of translational heading from optical flow. Journal of Experimental Psychology: Human Perception and Performance, 14, 644-660.

Waxman, A. M., \& Ullman, S. (1985). Surface structure and 3D motion from image flow: A kinematic analysis. International Journal of Robotics Research, 4, 72-94.

Whiteside, T. C., \& Samuel, G. D. (1970). Blur zone. Nature, 225, 94-95.

Received March 28, 1991

Revision received July 1, 1991

Accepted July 8, 1991 